\title{
Phase Transitions in Quantum Spin Systems with Isotropic and Nonisotropic Interactions
}

\author{
Freeman J. Dyson, ${ }^{1}$ Elliott H. Lieb, ${ }^{2}$ and Barry Simon ${ }^{2,3}$
}

Received November 1, 1976

We prove the existence of spontaneous magnetization at sufficiently low temperature, and hence of a phase transition, in a variety of quantum spin systems in three or more dimensions. The isotropic spin $1 / 2 x-y$ model and the Heisenberg antiferromagnet with spin $1,3 / 2, \ldots$ and with nearest neighbor interactions on a simple cubic lattice are included.

KEY WORDS : Phase transitions; Heisenberg ferromagnet.

\section{INTRODUCTION}

A basic subtlety in the study of statistical mechanics is the following: In nature, we observe abrupt changes in certain basic physical quantities, such as the magnetization of a magnet, but the statistical mechanics of systems with finitely many degrees of freedom is typically real analytic in all external variables. The resolution of this apparent paradox is that the abrupt changes are only approximately abrupt: True discontinuities only occur in the limit of an infinite system. For this reason, one must expect the problem of rigorously proving the existence of phase transitions to be a difficult one even for systems for which there is considerable numerical evidence or even a heuristic explanation for such a transition. In fact, until recently, the only general method available for directly proving the existence of phase transitions was Peierls' method, developed by Dobrushin ${ }^{(8)}$ and Griffiths ${ }^{(15)}$ on the basis of original ideas of Peierls. ${ }^{(35)}$ The method was developed originally

Research supported by U.S. National Science Foundation under grants GP-40768X (F.J.D.), MCS 75-21684 (E.H.L.), and GP-39048 (B.S.).

${ }^{1}$ Institute for Advanced Study, Princeton, New Jersey.

${ }^{2}$ Departments of Mathematics and Physics, Princeton University, Princeton, New Jersey.

${ }^{3}$ Alfred Sloan Fellow. 
for classical spin systems, but it has been extended by Ginibre ${ }^{(13)}$ and Robinson ${ }^{(39)}$ to treat highly anisotropic (Heisenberg) quantum models and by Glimm et al. ${ }^{(14)}$ to treat certain quantum field theories. The quantum spin systems were handled as perturbations of the classical (Ising) model, so that this method seems to be restricted to the highly anisotropic regime; the quantum field theories are treated by going to the Euclidean region, where they become essentially classical models. ${ }^{(19), 4}$

One way of describing the limitations of the Peierls argument is in terms of the broken symmetries that often accompany phase transitions-the simplest physical example is the occurrence of a spontaneous magnetization in some direction in the absence of an external field. In all cases of the Peierls argument accompanied by a broken symmetry, this symmetry has been a discrete (finite) symmetry group. Until recently, one has been unable to prove rigorously the existence of phase transitions in systems with a continuous symmetry group, such as the classical Heisenberg model ("classical" spins with values on the unit sphere in $\mathbb{R}^{3}$ ) or the quantum Heisenberg model. This situation has been changed by recent work of Fröhlich, Simon, and Spencer ${ }^{(12)}$ (henceforth FSS), who prove the existence of phase transitions in a variety of classical spin systems, including certain classical Heisenberg models. It is our goal in this paper to provide the first proof of phase transitions in any kind of quantum spin system with continuous symmetry-in particular, we will prove that such transitions occur in the spin-1 nearest neighbor, quantum Heisenberg antiferromagnet on a simple cubic lattice in three or more space dimensions. It is well known that a phase transition accompanied by a spontaneous magnetization cannot occur for this model in one or two dimensions. ${ }^{(21,28)}$

Since we use some of the ideas of FSS as an important element of our proof, it is useful to recall them. While FSS deal directly with infinite volume expectations, it is useful, for our purposes, to rephrase their results in terms of finite volume statements. Let $\Lambda$ be a parallelepiped in the simple $\nu$-dimensional cubic lattice $Z^{v}$ of the form

$$
\Lambda=\left\{\alpha \mid 0 \leqslant \alpha_{1} \leqslant L_{1}-1, \ldots, 0 \leqslant \alpha_{v} \leqslant L_{v}-1\right\}
$$

We shall refer to $\Lambda$ as the standard $L_{1} \times \cdots \times L_{\nu}$ box. In the classical model, one has a "spin" $\mathbf{S}_{\alpha}$ for each $\alpha \in \Lambda$, where $\mathbf{S}$ has three components $S^{(j)}$, $j=1,2,3$. These classical spins will be normalized by

$$
\mathbf{S}_{\alpha} \cdot \mathbf{S}_{\alpha} \equiv \sum_{j}\left(S_{\alpha}^{(j)}\right)^{2}=1
$$

and distributed according to the isotropic spherical distribution denoted by

${ }^{4}$ The Peierls argument has recently been extended to the entire anisotropic regime in the classical case ${ }^{(4 B)}$ and to a large regime for the quantum anisotropic ferromagnet. ${ }^{(49)}$ 
$d \lambda(\mathbf{S})$. The basic Hamiltonian is

$$
H=-\sum_{\alpha, i} \mathbf{S}_{\alpha} \cdot \mathbf{S}_{\alpha+\delta}
$$

where $i$ is summed from 1 to $\nu$, and $\alpha$ is summed over $\Lambda$. Here $\delta_{i}$ is the unit vector whose $i$ th component is 1 and we use the convention that if $\alpha_{i}=L_{i}-1$, then $\left(\alpha+\delta_{i}\right)_{i}=0$, i.e., $H$ has periodic boundary conditions. It is important that changing the boundary conditions does not affect the existence or nonexistence of a phase transition, so that the imposition of periodic boundary conditions is purely a matter of mathematical convenience. $H$ has each pair of nearest neighbors (in $\Lambda$, viewed as a torus) interacting once with coupling 1 . Since we will have the inverse temperature $\beta$ as a free variable, we do not add an additional factor $J$ in front of (2). We will occasionally use $\mathbf{S}_{\alpha} \cdot \mathbf{S}_{\alpha}=1$ to rewrite $H$ as

$$
H=\text { const }+\frac{1}{2} \sum_{\alpha, i}\left(S_{\alpha}-S_{\alpha+\delta_{i}}\right)^{2}
$$

The partition function $Z$ is defined by

$$
Z=\int \exp [-\beta H(\mathbf{S})] \prod_{\alpha \in \Lambda} d \lambda\left(\mathbf{S}_{\alpha}\right)
$$

and thermal expectations by

$$
\langle f(\mathbf{S})\rangle_{\Lambda, \beta}=Z^{-1} \int f(\mathbf{S}) \exp [-\beta H(\mathbf{S})] \prod_{\alpha \in \Lambda} d \lambda\left(\mathbf{S}_{\alpha}\right)
$$

Our translation of FSS is that their basic result is a proof that

$$
\lim _{\Lambda \rightarrow \infty}\left\langle\sum_{j=1}^{3}\left(|\Lambda|^{-1} \sum_{\alpha \in \Lambda} S_{\alpha}^{(j)}\right)^{2}\right\rangle_{\Lambda, \beta} \neq 0
$$

for $\beta$ sufficiently large. Intuitively, (4) corresponds to macroscopic fluctuations in the bulk magnetization (since $\left\langle S_{\alpha}^{(j)}\right\rangle=0$ by symmetry) and hence to the presence of a multiplicity of phases. We return to the question of relating (4) to other notions of phase transition at the conclusion of this section.

We introduce the Fourier variables $\hat{\mathbf{S}}_{\mathbf{p}}$ by

$$
\mathbf{S}_{\mathbf{p}}=\frac{1}{\sqrt{|\Lambda|}} \sum_{\alpha \in \Lambda}[\exp (-i \mathbf{p} \cdot \alpha)] \mathbf{S}_{\alpha}
$$

where $\mathbf{p}$ runs through the dual lattice $\Lambda^{*}$, i.e., $p_{j}=2 \pi n_{j} / L_{j} ; n_{j}=-\frac{1}{2} L_{j}+$ $1, \ldots, \frac{1}{2} L_{j}\left(L_{j}\right.$ even) or $-\frac{1}{2}\left(L_{j}-1\right), \ldots, \frac{1}{2}\left(L_{j}-1\right)\left(L_{j}\right.$ odd $)$. In terms of these Fourier variables, $H$ has the form

$$
H=\text { const }+\sum E_{\mathbf{p}} \hat{\mathbf{S}}_{\mathbf{p}} \cdot \hat{\mathbf{S}}_{-\mathbf{p}}
$$

where

$$
E_{\mathbf{p}}=\frac{1}{2} \sum_{|\delta|=1}[1-\exp (i \mathbf{p} \cdot \delta)]=\nu-\sum_{i=1}^{\nu} \cos p_{i} \geqslant 0
$$


and with the sum over $|\boldsymbol{\delta}|$ over the $2 \boldsymbol{\nu}$ vectors $\pm \boldsymbol{\delta}_{i}$. For a quantum system in which each spin has angular momentum $S, 2 S E_{\mathrm{p}}$ is the energy of a momentum-p spin wave. ${ }^{(4,9,10)}$ For later purposes we note that

$$
E_{\mathbf{p}} \sim|p|^{2} / 2 \text { for }|p| \text { small }
$$

In terms of the Fourier variables, (4) has the form

$$
\lim _{\Lambda \rightarrow \infty}|\Lambda|^{-1} g_{\mathbf{p}=0} \neq 0
$$

where

$$
g_{\mathbf{p}}=\left\langle\hat{\mathbf{S}}_{\mathbf{p}} \cdot \hat{\mathbf{S}}_{-\mathbf{p}}\right\rangle
$$

The FSS proof of $\left(4^{\prime}\right)$ comes from two bounds. The first is the Plancherel relation (which is completely trivial in this case since finite sums are involved), yielding a sum rule

$$
\frac{1}{|\Lambda|} \sum_{\mathbf{p} \in \Lambda^{*}} g_{\mathbf{p}}=\left\langle\left(\mathbf{S}_{\alpha}\right)^{2}\right\rangle=1
$$

(any value of $\alpha$ can be used). The second is the basic bound

$$
g_{\mathbf{p}} \leqslant 3 /\left(2 \beta E_{\mathbf{p}}\right) ; \quad \mathbf{p} \neq 0
$$

proven by FSS. The condition (10) has the physical interpretation that the average energy $E_{\mathbf{p}}\left\langle\hat{\mathbf{S}}_{\mathbf{p}}^{(j)} \cdot \hat{\mathbf{S}}_{-\mathbf{p}}^{(j)}\right\rangle$ per mode is dominated by its equipartition value of $\frac{1}{2} k T$ per degree of freedom, counting each value of $j$ as a separate degree of freedom. The bound (10) implies that

where

$$
\lim _{|\Lambda| \rightarrow \infty}|\Lambda|^{-1} \sum_{\mathbf{p} \neq 0} g_{\mathbf{p}} \leqslant(3 / 2 \beta) G_{v}(0)
$$

$$
G_{v}(0)=(2 \pi)^{-v} \int_{\left|p_{i}\right| \leq \pi}\left(E_{\mathrm{p}}\right)^{-1} d^{v} p
$$

and we have obtained the Fourier integral (11) as a limit of Fourier sums. $G_{v}(0)$ is finite when $\nu \geqslant 3$ by (7). The sum rule (9) implies that (4') holds so long as

$$
(3 / 2 \beta) G_{v}(0)<1
$$

i.e.,

$$
\beta>\frac{3}{2} G_{v}(0) \equiv \beta_{c}^{F S S}
$$

This method relies on the fact that the bound (10) and the sum rule (9) force a macroscopic occupation in the $\mathbf{p}=0$ mode. This is a kind of spin-wave Bose condensation. The above discussion is for the ferromagnet, but the same obviously applies to the antiferromagnet with $\mathbf{p}$ replaced by $(\pi, \ldots, \pi)-\mathbf{p}$ in suitable places.

To explain the problems that have to be overcome in extending the FSS results to the quantum case, we must describe the model. Let $S$ be a 
fixed number chosen from $1 / 2,1,3 / 2, \ldots$. Each site $\alpha \in \Lambda$ has associated with it a $(2 S+1)$-dimensional space $\mathscr{H}_{\alpha} \cong \mathbb{C}^{2 S+1}$ and three self-adjoint operators $\mathbf{S}_{\alpha}\left(S_{a}^{(j)}, j=1,2,3\right)$ obeying the usual commutation relations (summation convention used on Latin indices):

$$
\left[S_{\alpha}^{(j)}, S_{\alpha}^{(k)}\right]=i \epsilon_{j k l} S_{\alpha}^{(l)}
$$

However, (1) is replaced by

$$
\mathrm{S}_{\alpha}^{2}=\sum_{j}\left(S_{\alpha}^{(j)}\right)^{2}=S(S+1)
$$

Since $\operatorname{dim} \mathscr{H}_{\alpha}=2 S+1$, (13) and (1Q) essentially determine $\mathbf{S}_{\alpha}$ uniquely. In volume $\Lambda$, the basic Hilbert space is $\mathscr{H}_{\Lambda}=\otimes_{\alpha \in \Lambda} \mathscr{H}_{\alpha} \cong \mathbb{C}^{(2 S+1)|\Lambda|}$. We abuse notation by letting $S_{\alpha}$ stand for the triplet of operators on $\mathscr{H}_{\Lambda}$ that are the tensor product of 1 on each $\mathscr{H}_{\gamma}$ for $\gamma \neq \alpha$ and $\mathbf{S}_{\alpha}$ on $\mathscr{H}_{\alpha}$. The basic Hamiltonian is still given by (2) [or $\left(2^{\prime}\right)$ ] but now $Z$ and thermal expectations are given by

$$
\begin{aligned}
Z & =\operatorname{Tr}_{\mathscr{H}_{\Lambda}}\left[\exp \left(-\beta H_{\Lambda}\right)\right] \\
\langle A\rangle_{\Lambda, \beta} & =Z^{-1} \operatorname{Tr}\left[A \exp \left(-\beta H_{\Lambda}\right)\right]
\end{aligned}
$$

We still define operators $\widehat{\mathbf{S}}_{\mathbf{p}}$ by (5). Due to the commutation relations

$$
\left[S_{\alpha}^{(j)}, S_{\mathbf{\beta}}^{(k)}\right]=i \delta_{\alpha \beta} \epsilon_{j k i} S_{\alpha}^{(l)}
$$

one has that

$$
\left[\hat{S}_{\mathbf{p}}^{(j)}, \hat{S}_{\mathbf{q}}^{(k)}\right]=|\Lambda|^{-1 / 2}\left(i \epsilon_{j k l} \hat{S}_{\mathbf{p}+\mathbf{q}}^{(l)}\right)
$$

In particular, $\hat{S}_{\mathbf{p}}^{(j)}$ commutes with its adjoint $\hat{S}_{\mathbf{p}}^{(j)^{*}}=\hat{S}_{-\mathbf{p}}^{(j)}$ and, with the definition of $g_{\mathbf{p}}$ by (8), we have $g_{\mathbf{p}}=g_{-\mathbf{p}}$. The expression (2") still holds [we caution the reader that the constants in $\left(2^{\prime}\right)$ and $\left(2^{\prime \prime}\right)$ are different from those in the classical case]. The sum rule (9) is replaced by

$$
\frac{1}{|\Lambda|} \sum_{\mathbf{p} \in \Lambda^{*}} g_{\mathbf{p}}=S(S+1)
$$

The difficult problem to overcome is that (10) cannot be true in the quantum case! For, if (10) holds, then as $\beta \rightarrow \infty$ with $\Lambda$ fixed and finite, $g_{p}$ would approach zero and this, in turn, would imply that $\mathbf{S}_{\alpha} \cdot \mathbf{S}_{\gamma} \equiv f_{\alpha \gamma}$ approaches a constant as $\beta \rightarrow \infty$. But as $\beta \rightarrow \infty, f_{\text {ar }} \rightarrow S^{2}$ for $\alpha \neq \gamma$ and $S(S+1)$ for $\alpha=\gamma$. Therefore, because of the values of $f_{\alpha \gamma}$ in the ferromagnetic ground state, (10) is false in the quantum case. We believe that the following is true for the ferromagnet:

$$
g_{\mathbf{p}} \leqslant \sqrt{\frac{3}{2}} S \operatorname{coth}\left(\sqrt{\frac{2}{3}} S \beta E_{\mathbf{p}}\right)
$$

We will prove the analog of $(10 Q)$ for the antiferromagnet in a two-step 
process. First, we will prove (10) with $g_{\mathbf{p}}$ replaced by a "Duhamel two-point function," $b_{\mathrm{p}}$. Second, we will obtain bounds relating $b_{\mathrm{p}}$ and $g_{\mathrm{p}}$. The second step in the argument carries over to the ferromagnet. Our proof of the first part does not. We believe that the first bound will be true in that case, but are unable to prove it.

In Section 2, we discuss this Duhamel two-point function, which is not new (see the discussion in that section), and in Section 3 we prove a basic bound relating $b_{\mathrm{p}}$ to $g_{\mathrm{p}}$ (involving also the double commutator $\left.\sum_{j=1}^{3}\left[\left[\hat{S}_{\mathbf{p}}^{(j)}, H\right], \hat{S}_{-\mathbf{p}}^{(j)}\right]\right)$. Section 4 contains a proof of the analog of $(10)$ with $g_{\mathbf{p}}$ replaced by $b_{\mathbf{p}}$. In Section 5 we put everything together to prove a phase transition in the sense of (4). In Sections 6 and 7 and the appendices we discuss the Heisenberg antiferromagnet and additional results.

We should emphasize here that certain aspects of our argument are very general. The bounds in Section 3 are "operator theoretic" in the sense that they depend on no special properties of the Hamiltonian. As we will explain, we believe that the bounds in Section 4 have an extension to any antiferromagnetic quantum lattice system, but this part of our proof only works for nearest neighbor interactions on a simple cubic lattice (or a rather small class of other lattices that does not include face- or body-centered cubic lattices). In any event, the bounds in Section 4 depend neither on algebraic properties of the spins nor on the norms of the spin operators. It is only in combining the bounds from Sections 3 and 4 with a sum rule of the type of $(9 Q)$ that these detailed properties of the spin enter. It is here that the $S$ dependence of our critical temperature bounds arises.

We also note that modulo a factor of $3 / 2$, the bounds that would follow if $(10 Q)$ could be proved for the ferromagnet, have the interpretation of making rigorous certain elements of spin-wave theory..$^{(4,9,10)}$ We discuss this point further in Appendix B.

Finally, we turn to relating the criterion (4) for phase transitions to other criteria. This is a problem discussed already by Griffiths ${ }^{(16)}$ (see Hepp and Lieb ${ }^{(20)}$ for related results). Let us begin by giving an abstract version of Griffiths' main theorem and corollary (Ref. 16, §II.f) motivated by the form of the results given in the appendix to Ref. 20 .

To motivate the following theorem, it is useful to think of $n$ as parametrizing the size of a magnetic system, $x$ as the magnetization, $y$ as a magnetic field, and $\alpha$ as the partition function.

Theorem 1.1 (Griffiths ${ }^{(16)}$ ). Let $\mu_{n}$ be a sequence of probability measures (i.e., $\int d \mu_{n}=1$ ) on the real line such that, for $y$ in an interval $[-c, c]$ about 0 ,

$$
\alpha(y)=\lim _{n \rightarrow \infty} \frac{1}{n} \ln \int e^{y x} d \mu_{n}(x)
$$


exists (and is finite). We assume $c>0$. Let

$$
a_{ \pm}= \pm \lim _{y \downarrow 0}\left\{y^{-1}[\alpha( \pm y)-\alpha(0)]\right\}
$$

(which exists since $\alpha$ is convex). Let $\delta>0$ be fixed. Then

$$
\varlimsup\left[\int_{\left(a_{+}+\delta\right) n}^{\infty} d \mu_{n}(x)\right]^{1 / n}<1 ; \quad \varlimsup\left[\int_{-\infty}^{\left(a_{-}-\delta\right) n} d \mu_{n}(x)\right]^{1 / n}<1
$$

Proof. We prove the first statement. Let

$$
b_{n}=\int_{\left(a_{+}+\delta\right) n}^{\infty} d \mu_{n}(x)
$$

If the first statement is false, then $\overparen{\lim }(1 / n) \ln b_{n} \geqslant 0$. Clearly, for $y \geqslant 0$

$$
\int e^{y x} d \mu_{n}(x) \geqslant b_{n} e^{n\left(a_{+}+\delta\right) y}
$$

so that, if $\overline{\lim }(1 / n) \ln b_{n} \geqslant 0$,

$$
\alpha(y) \geqslant\left(a_{+}+\delta\right) y
$$

Since $\alpha(0)=0$,

$$
\lim _{y \downarrow 0} y^{-1}[\alpha(y)-\alpha(0)] \geqslant a_{+}+\delta
$$

which is impossible.

This theorem says that

$$
\int_{\left(a_{-}-\delta\right) n}^{\left(a_{+}+\delta\right) n} d \mu_{n}(x)
$$

goes to 1 with exponentially small error.

Corollary 1.1. Under the hypothesis of Theorem 1.1, if $a_{+}=a_{-}=a$ [i.e., if $\alpha(y)$ is differentiable at $y=0$ ], then for functions $f$ obeying $|f(x)| \leqslant$ $A e^{B|x|}$ for some $B<c$ (defined in Theorem 1.1)

$$
\lim _{n \rightarrow \infty} \int f(x / n) d \mu_{n}(x)=f(a)
$$

Remark. For $f(x)=x^{k}$, this is a result of Hepp and Lieb. ${ }^{(20)}$

Proof. For any $f$ that is bounded, this follows immediately from the theorem. Fix $\beta>0$. Then

$$
\begin{aligned}
\int_{x \geq \beta n}|f(x / n)| d \mu_{n}(x) & \leqslant A \int_{x \geq \beta n} e^{B x / n} d \mu_{n}(x) \\
& \leqslant A e^{(B-c) \beta} \int e^{c x / n} d \mu_{n}(x) \\
& \leqslant A e^{(B-c) \beta}\left[\int e^{c x} d \mu_{n}(x)\right]^{1 / n}
\end{aligned}
$$


by Hölder's inequality. Thus

$$
\varlimsup \int_{|x| \geqslant \beta n}|f(x / n)| d \mu_{n}(x) \leqslant A e^{(B-c) \beta}[\alpha(c)+\alpha(-c)]
$$

so that, given $\epsilon$, we can find $\beta_{0}$ such that

$$
\varlimsup_{n \rightarrow \infty} \int_{|x| \geq \beta_{0} n}|f(x / n)| d \mu_{n}(x) \leqslant \epsilon
$$

Since $f(\cdot / n)$ is bounded on $\left(-\beta_{0} n, \beta_{0} n\right)$, we can use the remark at the beginning of the proof to conclude that

$$
f(a)-\epsilon \leqslant \underline{\lim } \int f(x / n) d \mu_{n}(x) \leqslant \varlimsup \int f(x / n) d \mu_{n}(x) \leqslant f(a)+\epsilon
$$

Since $\epsilon$ is arbitrary, the result follows.

Corollary 1.2. Under the hypotheses of Theorem 1.1, for any function $f$ obeying $|f(x)| \leqslant A e^{B|x|}$ for some $B<c$

$$
\varlimsup_{n \rightarrow \infty} \int f(x / n) d \mu_{n}(x) \leqslant \max _{a_{-} \leq y \leq a_{+}} f(y)
$$

In particular, if $f(y)=y^{2 k}\left(k\right.$ an integer) and $a_{-}=-a_{+}$, then

$$
a_{+} \geqslant \varlimsup_{n \rightarrow \infty}\left[\int(x / n)^{2 k} d \mu_{n}(x)\right]^{1 / 2 k}
$$

Remark. The case $f(y)=y^{2 k}$ with $x / n$ bounded on supp $\mu_{n}$ is the main result of Griffiths, ${ }^{(16)}$ whose proof is abstracted above.

Proof. For $f$ bounded, the result follows from Theorem 1.1. The general case follows as in Corollary 1.1.

Following Griffiths, ${ }^{(16)}$ we can apply Corollary 1.2 to prove that longrange order in the sense of (4) implies a spontaneous magnetization. Let $H_{\Lambda}$ be the Hamiltonian of a system in a box with periodic boundary conditions (but with no restriction on the form of the interaction). Let $A_{\alpha}$ be an operator at site $\alpha$. Define $m(A)$ by

$$
m(A)=\lim _{\mu \downarrow 0} \frac{d}{d \mu}\left(\lim _{\Lambda \rightarrow \infty}|\Lambda|^{-1} \ln \left\{\operatorname{Tr}\left[\exp \left(-\beta H_{\Lambda}+\mu \sum A_{\alpha}\right)\right]\right\}\right)
$$

As an immediate consequence of Corollary 1.2, we have the following result:

Theorem 1.2. Under the above conditions, if $\sum_{\alpha \in \Lambda} A_{\alpha}$ commutes with $H_{\Lambda}$, then

$$
m(A) \geqslant \overline{\lim }_{\Lambda \rightarrow \infty}\left\langle\left(|\Lambda|^{-1} \sum_{\alpha \in \Lambda} A_{\alpha}\right)^{2 k}\right\rangle_{\Lambda}^{1 / 2 k}
$$


In particular, if there is a unitary operator leaving $H_{\Lambda}$ invariant but taking $A_{\alpha}$ to $-A_{\alpha}$ and if the right side of (14) is nonzero for some $k$, then there is a phase transition in the sense that $\lim _{\Lambda \rightarrow \infty}|\Lambda|^{-1} \ln \left\{\operatorname{Tr}\left[\exp \left(-\beta H_{\Lambda}+\mu \sum A_{\alpha}\right)\right]\right\}$ is nondifferentiable at $\mu=0$.

Remark 1. It is well known ${ }^{(41)}$ that the nondifferentiability of the free energy implies multiple "phases" in the sense of several equilibrium states.

Remark 2. If $\operatorname{Tr}\left[\exp \left(-\beta H_{\Lambda}+\mu \sum A_{\alpha}\right)\right]$ is replaced by $\operatorname{Tr}\left[\exp \left(-\beta H_{\Lambda}\right)\right.$ $\left.\exp \left(\mu \sum A_{q}\right)\right]$, the commutation condition is not needed; the commutation condition is only used to obtain the physically relevant object. The commutativity unfortunately fails in several cases of interest, notably the $x-y$ and the antiferromagnetic models. In Section 5, after Theorem 5.2, we develop a different strategy for proving the existence of a phase transition in the noncommutative case, and apply it there to the $x-y$ model. In Section 6 we use it again for the antiferromagnet.

Theorem 1.2 with $k=1$ shows that (4) implies there is a phase transition in general systems, but one should expect that in the isotropic Heisenberg model it yields a lower bound on $m\left(S^{(3)}\right)$ which is too small by a factor of three. For, in the isotropic model, $\left\langle\left(\sum S_{a}^{(3)}\right)^{2}\right\rangle=\frac{1}{3}\left\langle\left|\sum \mathbf{S}_{\alpha}\right|^{2}\right\rangle$ by symmetry, but as soon as an external field in the other direction is turned on, the LeeYang theorem ${ }^{(2)}$ implies that $\left\langle\left(|\Lambda|^{-1} \sum S_{\alpha}^{(i)}\right)^{2}\right\rangle \rightarrow 0$ as $|\Lambda| \rightarrow \infty$ for $i=1,2$. This can be remedied by the use of some angular momentum theory.

Theorem 1.3. In the isotropic Heisenberg model, the spontaneous magnetization $m\left(S^{(3)}\right)$ obeys

$$
m\left(S^{(3)}\right)^{2} \geqslant \lim _{\Lambda \rightarrow \infty}\left\langle\left.\left.|| \Lambda\right|^{-1} \sum_{\alpha \in \Lambda} S_{\alpha}\right|^{2}\right\rangle_{\Lambda}=3 \lim _{\Lambda \rightarrow \infty}\left\langle\left.\left.|| \Lambda\right|^{-1} \sum S_{\alpha}^{(3)}\right|^{2}\right\rangle
$$

Remark. The only restriction on the interaction is its isotropy in spin space, i.e., that $H$ commutes with simultaneous rotations of all spins.

Proof. Let $J_{(i)}=\sum_{\alpha \in \Lambda} S_{\alpha}^{(i)}$ and define

$$
|J|=\left(J_{(1)}^{2}+J_{(2)}^{2}+J_{(3)}^{2}+\frac{1}{4}\right)^{1 / 2}-\frac{1}{2}
$$

Letting

$$
a(j, k)=(2 j+1)^{-1} \sum_{n=-j}^{j} n^{k}
$$

we have by the isotropy of $H$ that

$$
\left\langle J_{(3)}^{2 k}\right\rangle=\sum_{j} a(j, 2 k) \operatorname{prob}(|J|=j)
$$


For simplicity of notation, consider the case where $|\Lambda|$ is even, so that $|J|$ has integral eigenvalues. For $j$ integral

$$
\begin{aligned}
a(j, 2 k) & \geqslant \frac{2}{2 j+1} \sum_{n=1}^{j} n^{2 k} \geqslant \frac{2}{2 j+1} \int_{0}^{j} x^{2 k} d x \\
& =\frac{j^{2 k}}{2 k+1} \frac{2 j}{2 j+1} \geqslant \frac{2}{3} \frac{j^{2 k}}{2 k+1}, \quad j=0,1, \ldots ; \quad k \geqslant 1
\end{aligned}
$$

Therefore, for $k \geqslant 1$,

$$
\left\langle J_{(3)}^{2 k}\right\rangle \geqslant \frac{2}{3(2 k+1)}\left\langle|J|^{2 k}\right\rangle \geqslant \frac{2}{3(2 k+1)}\left\langle|J|^{2}\right\rangle^{k}
$$

Using the definition of $|J|$, it is not hard to see that

$$
\lim _{\Lambda \rightarrow \infty}\left\langle\left.\left.|| \Lambda\right|^{-1} \sum_{\alpha \in \Lambda} \mathbf{S}_{\alpha}\right|^{2}\right\rangle=\lim _{\Lambda \rightarrow \infty}|\Lambda|^{-2}\left\langle|J|^{2}\right\rangle
$$

so that the result follows by taking $k \rightarrow \infty$ in (15) and applying Theorem 1.2 .

\section{THE DUHAMEL TWO-POINT FUNCTION}

For quantum systems in finite volume with partition function $Z=$ $\operatorname{Tr}\left(e^{-\beta H}\right)$, we define the Duhamel two-point function (DTF) by

$$
(A, B)=Z^{-1} \int_{0}^{1} \operatorname{Tr}\left(e^{-x \beta H} A e^{-(1-x) B H} B\right) d x
$$

One expression of the naturalness of this object is that it has been introduced and discussed by a variety of authors, e.g., Bogoliubov, ${ }^{(5)} \mathrm{Kubo}^{(24)}$ Hohenberg, ${ }^{(21)}$ Mermin and Wagner, ${ }^{(28)}$ Mori, ${ }^{(29)}$ Naudts et al. ${ }^{(30,31)}$ Powers, ${ }^{(36)}$ and Roepstorff. ${ }^{(40)}$ Fröhlich ${ }^{(46)}$ has independently noted that it is likely to be useful in finding quantum generalizations of $(10)$. We warn the reader that (16) may differ by factors of $\beta$ and by adjoints from the conventions of the above authors.

The name we have chosen comes from the fact that $(1 / 2) \mu^{2}(A, A) Z$ is the second-order term in a perturbation expansion, first derived by Duhamel, for $\operatorname{Tr}[\exp (-\beta H+\mu A)]$, i.e.,

$$
(A, B) Z=\frac{\partial^{2}}{\partial \mu \partial \lambda} \operatorname{Tr}[\exp (-\beta H+\mu A+\lambda B)]
$$

From (17), or from the definition (16), it is obvious that

$$
(A, B)=(B, A)
$$


so that, in particular, if $A=A_{\tau}+i A_{i}$ with $A_{r}^{*}=A_{r}, A_{i}^{*}=A_{i}$, then

$$
\left(A^{*}, A\right)=\left(A_{r}, A_{r}\right)+\left(A_{i}, A_{i}\right)
$$

If $\langle B\rangle_{\mu} \equiv\{\operatorname{Tr}[\exp (-\beta H+\mu A)]\}^{-1} \operatorname{Tr}[B \exp (-\beta H+\mu A)]$, then

$$
\left.\frac{\partial\langle B\rangle_{\mu}}{\partial \mu}\right|_{\mu=0}=(A, B)-\langle A\rangle\langle B\rangle=(A-\langle A\rangle, B-\langle B\rangle)
$$

so that, for example, any kind of generalization of Griffiths' second inequality ${ }^{(17)}$ to quantum systems would involve the DTF and not the thermal two-point function, $\langle A B\rangle \equiv Z^{-1} \operatorname{Tr}[A B \exp (-\beta H)]$. Unlike $(A, B)$, $\langle A B\rangle$ is not symmetric in $A$ and $B$. If $H$ has a complete set of eigenfunctions $\phi_{i}$ with $H \phi_{i}=\epsilon_{i} \phi_{i}$ and $a_{i j}=\left(\phi_{i}, A \phi_{j}\right), b_{i j}=\left(\phi_{i}, B \phi_{j}\right)$, then

$$
\begin{aligned}
(A, B) & =Z^{-1} \int_{0}^{1} \sum_{i, j} a_{i j} b_{j i} e^{-x \beta \epsilon_{i}} e^{-(1-x) \beta \epsilon_{j}} d x \\
& =(\beta Z)^{-1} \sum_{i, j} a_{i j} b_{j i}\left(e^{-\beta \epsilon_{i}}-e^{-\beta \epsilon_{j}}\right) /\left(\epsilon_{j}-\epsilon_{i}\right)
\end{aligned}
$$

Up to factors of $\beta$ and $Z$, the reader will recognize the formula from Ruelle's book $^{(41)}$ for the inner product he uses in his proof of Bogoliubov's inequality. From either (21) or directly from (16) [writing $\operatorname{Tr}\left(e^{-x \beta H} A^{*} e^{-(1-x) \beta H} A\right)=$ $\operatorname{Tr}\left(C_{x}{ }^{*} C_{x}\right)$, with $\left.C_{x}=e^{-(1-x) \beta H / 2} A e^{-x \beta H / 2}\right]$, one sees that

$$
\left(A^{*}, A\right) \geqslant 0
$$

so that we have a Schwarz inequality [using $\left.\left(A^{*}, A\right)=\left(A, A^{*}\right)\right]$

$$
|(A, B)| \leqslant\left(A^{*}, A\right)^{1 / 2}\left(B^{*}, B\right)^{1 / 2}
$$

Since the thermal expectation $\langle A B\rangle$ is not symmetric in $A$ and $B$, it is not clear which "two-point function" is closest to its classical analog. Some insight into this is obtained by looking at harmonic oscillators with variable $\hbar$. Let $A=a \sqrt{\hbar}$, with $a$ being the "usual" creation operator. Then $H=\omega A^{*} A$. As can be seen by direct calculation (Appendix $\left.\mathrm{B}\right),\left(A^{*}, A\right)$ is independent of $\hbar$ but $(1 / 2)\left\langle A^{*} A+A A^{*}\right\rangle$ is not. In this sense, $\left(A^{*}, A\right)$ is the most classical two-point function that can be constructed, and from this point of view it is not surprising that the classical bound (10) also holds for the corresponding DTF.

From knowledge of the DTF for all pairs $A, B$, one can recover the thermal expectations via the trivial identity

$$
\langle A\rangle=(A, 1)
$$

Conversely, one can recover the DTF in finite volume from thermal expectations and the action of the group of time automorphisms

$$
\alpha_{t}(A)=e^{i t H} A e^{-i t H}
$$


To do so, one can use the function

$$
f(z)=\left\langle B \alpha_{z \beta}(A)\right\rangle
$$

which is defined a priori for $z$ real. It has an analytic continuation to the strip $\operatorname{Im} z \leqslant 1$ with

$$
f(z+i)=\left\langle A \alpha_{-z \beta}(B)\right\rangle
$$

and

$$
(A, B)=\int_{0}^{1} f(i x) d x
$$

This connection between the DTF and the KMS boundary condition is not new; see, e.g., Refs. 31,36 , and 40 . It leads quite easily ${ }^{(40)}$ to the bound

$$
\left(A^{*}, A\right) \leqslant \frac{1}{2}\left\langle A^{*} A+A A^{*}\right\rangle
$$

for, since $f$ with $B=A^{*}$ is analytic, the three-line lemma implies that

$$
|f(i x)| \leqslant a^{x} b^{1-x} \leqslant x a+(1-x) b
$$

where $a=\sup _{z \in \mathbb{R}}|f(i+z)| ; b=\sup _{z \in \mathbb{R}}|f(z)|$. But, for $z \in \mathbb{R},|f(z+i)| \leqslant$ $\left|\left\langle A \alpha_{-z \beta}\left(A^{*}\right)\right\rangle\right| \leqslant\left\langle A A^{*}\right\rangle$ and $|f(z)| \leqslant\left\langle A^{*} A\right\rangle$ by the Schwarz inequality. Thus $a=\left\langle A A^{*}\right\rangle$ and $b=\left\langle A^{*} A\right\rangle$, whence (25) follows from (26) by integration. The connection with the KMS boundary condition is also the key to extending many of the above considerations to infinite volume. ${ }^{(31)}$

The remainder of this section is not needed for the argument of the paper but is included to give the reader a source for all the main lore about the DTF. First, we want to prove (following Powers ${ }^{(36)}$ ) Bogoliubov's inequality ${ }^{(28)}$ using the representation (16). We will use (22'), (25), and the formula

$$
\langle[A, B]\rangle=([A, \beta H], B)
$$

Now, (27) follows by noting that

$$
\operatorname{Tr}\left(e^{-x \beta H}[A, \beta H] e^{-(1-x) \beta H} B\right)=\frac{d}{d x} \operatorname{Tr}\left(e^{-x \beta H} A e^{-(1-x) \beta H} B\right)
$$

so that (16) can be directly integrated. Thus

$$
\begin{aligned}
|\langle[A, B]\rangle|^{2} & \leqslant|([A, \beta H], B)|^{2} \\
& \leqslant\left(B^{*}, B\right)\left([A, \beta H]^{*},[A, \beta H]\right) \\
& \leqslant\left(\frac{1}{2}\left\langle B^{*} B+B B^{*}\right\rangle\right)\left(\left[A^{*}, \beta H\right],[\beta H, A]\right) \\
& \leqslant \frac{1}{2}\left\langle B^{*} B+B B^{*}\right\rangle\left\langle\left[A^{*},[\beta H, A]\right]\right\rangle
\end{aligned}
$$


which is Bogoliubov's inequality,

$$
|\langle[A, B]\rangle|^{2} \leqslant\left\langle\left[A^{*},[\beta H, A]\right]\right\rangle\left(\frac{1}{2}\left\langle B^{*} B+B B^{*}\right\rangle\right)
$$

We would like to note two properties of the thermal expectation of the double commutator. First, $\left\langle\left[A^{*},[\beta H, A]\right]\right\rangle \geqslant 0$. This follows from (27) or by an eigenfunction expansion

$$
\begin{aligned}
\left\langle\left[A^{*},[\beta H, A]\right]\right\rangle & =\beta \sum_{i, j}\left(\left|a_{i j}\right|^{2}+\left|a_{j i}\right|^{2}\right)\left(\epsilon_{i}-\epsilon_{j}\right) e^{-\beta \epsilon_{j}} \\
& =\frac{1}{2} \beta \sum_{i, j}\left(\left|a_{i j}\right|^{2}+\left|a_{j i}\right|^{2}\right)\left(\epsilon_{i}-\epsilon_{j}\right)\left(e^{-\beta \epsilon_{j}}-e^{\beta \epsilon_{i}}\right) \geqslant 0
\end{aligned}
$$

since $(x-y)\left(e^{-y}-e^{-x}\right) \geqslant 0$ for all $x$ and $y$. Second, if $A=A_{r}+i A_{i}$ with $A_{r}$ and $A_{i}$ self-adjoint, then

$$
\left\langle\left[A^{*},[\beta H, A]\right]\right\rangle=\left\langle\left[A_{r},\left[\beta H, A_{r}\right]\right]\right\rangle+\left\langle\left[A_{i},\left[\beta H, A_{i}\right]\right]\right\rangle
$$

Finally, we want to say a few words about the infinite-volume DTF.

Proposition 2.1. If the finite-volume DTFs converge through some sequence of volumes, so do the ordinary thermal expectations. Conversely, if the ordinary finite-volume thermal expectations converge and the finitevolume time automorphisms converge, so do the DTFs.

Proof. The proposition is a consequence of formulas (23) and (24) relating ordinary thermal expectations and DTFs.

Remark 1. In infinite volume, one cannot define the DTF by (16), but (24) still holds. From this realization ${ }^{(31)}$ follow all the relations we use in the proof of the next section, so our results there hold directly in any KMS state without reference to proving the result in finite volume and making a limiting argument.

Remark 2. For discussion of the limit of finite-volume time automorphisms for spin systems, see Streater, ${ }^{(44)}$ Robinson, ${ }^{(38)}$ and Lieb and Robinson. ${ }^{(27)}$

Definition. We say the (infinite-volume) DTF clusters if and only if $\lim _{|\mathbf{a}| \rightarrow \infty}\left(A, \tau_{\mathbf{a}}(B)\right)=\langle A\rangle\langle B\rangle$, where $\tau_{\mathbf{a}}$ is the space translation.

Theorem 2.1. The DTF clusters if the ordinary thermal function clusters, i.e., $\lim _{|\mathbf{a}| \rightarrow \infty}\left\langle A \tau_{\mathbf{a}}(B)\right\rangle=\langle A\rangle\langle B\rangle$.

Proof. Suppose that the thermal function clusters. Let $f_{\mathbf{a}}(z)=$ $\left\langle\tau_{\mathrm{a}}(B) \alpha_{z \beta}(A)\right\rangle$. Then, by hypotheses, $f_{\mathrm{a}}(z)-\langle A\rangle\langle B\rangle \rightarrow 0$ as $\mathbf{a} \rightarrow \infty$ for $z$ real or $z=i+$ real. Moreover, for all $z$ and $\mathbf{a},\left|f_{\mathbf{a}}(z)\right| \leqslant\|A\|\|B\|$. Thus, by a simple complex variable argument, $f_{\mathrm{a}}(z) \rightarrow\langle A\rangle\langle B\rangle$ for all $z$, so $\left(A, \tau_{\mathrm{a}}(B)\right)=$ $\int_{0}^{1} f_{\mathbf{a}}(i x) d x \rightarrow\langle A\rangle\langle B\rangle$. 


\section{LOWER BOUNDS ON THE DUHAMEL TWO-POINT FUNCTION}

As explained in the introduction, the natural quantum extension of (10) involves the DTF, while the sum rule involves the thermal two-point function. To put the two together, we need a lower bound on the DTF in terms of the thermal two-point function. We have already seen that the easy bound (25) goes in the other direction. The lower bound will involve the function $f$ from $[0, \infty)$ to $[0,1)$ defined implicitly by the relation

$$
f(x \tanh x)=x^{-1} \tanh x
$$

and plotted in Fig. 1 (for which we thank J. F. Barnes). We will need:

Lemma 3.1. The function $f$ given by (30) is convex.

This lemma is proved in Appendix A. By absorbing $\beta$ into $H$ and adding a constant to $H$ so that $\operatorname{Tr}\left(e^{-H}\right)=1$, we can always deal with thermal expectations defined by $\langle B\rangle=\operatorname{Tr}\left(B e^{-H}\right)$. We now define

$$
\begin{aligned}
& g(A)=\frac{1}{2}\left\langle A^{*} A+A A^{*}\right\rangle=\frac{1}{2} \operatorname{Tr}\left[\left(A^{*} A+A A^{*}\right) e^{-H}\right] \\
& b(A)=\left(A^{*}, A\right)=\int_{0}^{1} \operatorname{Tr}\left(A^{*} e^{-x H} A e^{-(1-x) H}\right) d x \\
& c(A)=\left\langle\left[A^{*},[H, A]\right]\right\rangle=\operatorname{Tr}\left(\left[A^{*},[H, A]\right] e^{-H}\right)
\end{aligned}
$$

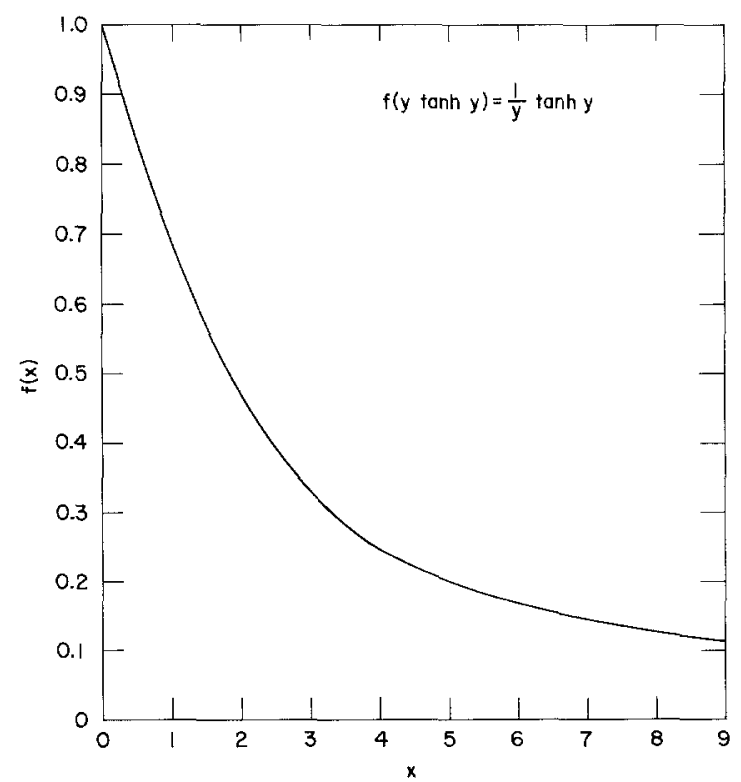

Fig. 1. A graph of the convex function $f$ defined for $x \geqslant 0$ by $f(y \tanh y)=y^{-1} \tanh y$. This function appears in the inequality, Theorem 3.1, between the Duhamel and ordinary two-point functions. For $x \geqslant 6, f(x)=x^{-1}$ to five-place accuracy. (Values computed by J. F. Barnes.) 
The following basic bound is a generalization and improvement of a bound of Roepstorf ${ }^{(40)}$-we shall discuss the precise connection after its proof. ${ }^{5}$

Theorem 3.1. Let $f$ be the function given by (30). Then for any $A$ and self-adjoint $H$

$$
b(A) \geqslant g(A) f(c(A) / 4 g(A))
$$

Proof. Let

$$
h(x)=\operatorname{Tr}\left(A^{*} e^{-x H} A e^{-(1-x) H}\right)
$$

Then

$$
g(A)=\frac{1}{2}[h(0)+h(1)], \quad b(A)=\int_{0}^{1} h(x) d x, \quad c(A)=h^{\prime}(1)-h^{\prime}(0)
$$

Moreover, if $H \phi_{n}=E_{n} \phi_{n}$ is an eigenfunction expansion of $H$,

$$
h(x)=\sum_{n, m}\left|a_{n m}\right|^{2} e^{-E_{m}} e^{+x\left(E_{m}-E_{n}\right)}
$$

so that $h$ is the Laplace transform of a positive measure. As a result, inequality (34) clearly follows from Proposition 3.1 below.

Proposition 3.1. Let $f$ be the function given by (30). Let

$$
h(x)=\int e^{x t} d \mu(t)
$$

for some positive measure $\mu$. Then $b \geqslant g f(c / 4 g)$, where $b=\int_{0}^{1} h(x) d x$, $g=\frac{1}{2}[h(0)+h(1)], c=h^{\prime}(1)-h^{\prime}(0)$.

Proof. Let $d \nu$ be the measure

$$
d v(t)=\frac{1}{2}\left(e^{t}+1\right) d \mu(t)
$$

Then

$$
\begin{aligned}
& b=\int t^{-1}\left(e^{t}-1\right) d \mu(t)=\int \frac{2}{t} \tanh \frac{t}{2} d \nu(t) \\
& g=\int \frac{1}{2}\left(e^{t}+1\right) d \mu(t)=\int d \nu(t) \\
& c=\int t\left(e^{t}-1\right) d \mu(t)=4 \int \frac{1}{2} t \tanh \frac{1}{2} t d \nu(t)
\end{aligned}
$$

${ }^{5}$ Unfortunately we were unaware that Falk and $\mathrm{Bruch}^{(47)}$ had previously proved our Theorem 3.1 [middle inequality in their Eq. (8)] and our Theorem A.4 [first inequality in their Eq. (8)]. 
Since $d \omega(t)=g^{-1} d v(t)$ is a probability measure and $f$ is convex (Lemma 3.1), we have by Jensen's inequality

$$
\begin{aligned}
g f(c / 4 g) & =g f\left(\int \frac{1}{2} t \tanh \frac{1}{2} t d \omega\right) \\
& \leqslant g \int f\left(\frac{1}{2} t \tanh \frac{1}{2} t\right) d \omega \\
& =g \int(2 / t) \tanh \frac{1}{2} t d \omega=b
\end{aligned}
$$

Remark 1. The strict convexity of $f$ and the above proof make it clear that equality holds in (34) if and only if $d \mu$ is a measure concentrated at a single point. From (35), this clearly holds if $A$ is a creation operator and $H$ is the Hamiltonian of a harmonic oscillator; this also follows from explicit calculation (see Appendix B).

Remark 2. Since one can shift the position of the point mass in Remark 1 by replacing the harmonic oscillator Hamiltonian $H$ by $\omega H$, we can adjust $\omega$ so that $c / 4 g$, the argument of $f$, has any preassigned positive value. Since equality always holds in (34) for the harmonic oscillator, it follows that the function $f$ in (34) is the best possible.

Remark 3. In Ref. 40, Roepstorff proved a bound of the form of (34) with two changes. First, he required that $A=A^{*}$, and second, he used the function

$$
f_{R}(x)=x^{-1}\left(1-e^{-x}\right)
$$

in place of $f$. Since one can prove directly that

$$
f(x) \geqslant f_{R}(x), \quad \text { all } x
$$

(see Appendix A), our inequality is stronger in two ways. Since we wish to use the inequality for $A^{\prime}$ 's with $A \neq A^{*}$, the former change is more significant. Roepstorff's inequality, had he proved it for all $A$, would lead to a phase transition in all systems where we prove one, albeit only at a lower transition temperature than ours.

Remark 4. As we will see shortly, there is an abstract method of extending inequalities like (34) or Roepstorff's inequality from Hermitian $A$ to all $A$. Given this fact, (37) follows also from the best possible nature of our inequalities. (See Remark 2 above.)

Lemma 3.2. Let $c_{1}, \ldots, c_{n}, b_{1}, \ldots, b_{n}, g_{1}, \ldots, g_{n}$ be real numbers and let $c=\sum_{i=1}^{n} c_{i}, b=\sum_{i=1}^{n} b_{i}, g=\sum_{i=1}^{n} g_{i}$. Assume that $g_{i} \geqslant 0$ and $g>0$, and 
$g_{i}=0 \Rightarrow b_{i} \geqslant 0$ and $c_{i}=0$. Let $F$ be any convex function on an interval $I$ that includes all $c_{i} / 4 g_{i}$ where $g_{i}>0$ and such that

$$
b_{i} \geqslant g_{i} F\left(c_{i} / 4 g_{i}\right)
$$

when $g_{i}>0$. Then $c / 4 g$ lies in the interval $I$ and

$$
b \geqslant g F(c / 4 g)
$$

Proof. Let $p_{i} \equiv g_{i} / g \geqslant 0$, so that $\sum_{i=1}^{n} p_{i}=1$. Then

$$
c / 4 g=\sum^{\prime}\left(c_{i} / 4 g_{i}\right) p_{i}
$$

where $\Sigma^{\prime}$ means the summation over those $i$ with $p_{i}>0$. Thus $c / 4 g$ is a convex combination of points in $I$ and is therefore in $I$. Moreover,

$$
\begin{aligned}
F(c / 4 g) & =F\left(\sum^{\prime} p_{i}\left(c_{i} / 4 g_{i}\right)\right) \\
& \leqslant \sum^{\prime} p_{i} F\left(c_{i} / 4 g_{i}\right) \quad \text { (by convexity) } \\
& =g^{-1} \sum^{\prime} g_{i} F\left(c_{i} / 4 g_{i}\right) \leqslant b / g
\end{aligned}
$$

Corollary 3.1. An estimate of the form (34) need only be proved for $A$ self-adjoint (and in particular, Roepstorff's estimate for $A=A^{*}$ implies his estimate for all $A$ ).

Remark. Since our estimate is proved directly for all $A$ and is best possible, this corollary is of academic interest only.

Proof. Let $A=A_{r}+i A_{i}$ with $A_{r}=A_{r}{ }^{*}, A_{i}=A_{i}{ }^{*}$. We have already seen [Eqs. (19) and (29)] that $c(A)=c\left(A_{r}\right)+c\left(A_{i}\right)$, and $b(A)=b\left(A_{r}\right)+b\left(A_{i}\right)$. That $g(A)=g\left(A_{r}\right)+g\left(A_{i}\right)$ is trivial.

Corollary 3.2. Let $\mathbf{A}=\left(A_{i}, \ldots, A_{n}\right)$ be an $n$-tuple of operators, let $g(\mathbf{A})=\sum_{i} g\left(A_{i}\right)$, etc., and let $f$ be given by (30). Then

$$
b(\mathbf{A}) \geqslant g(\mathbf{A}) f(c(\mathbf{A}) / 4 g(\mathbf{A}))
$$

Remark. One can also prove this corollary by mimicking the proof of Theorem 3.1, using the fact that the sum of Laplace transforms of positive measures is again a positive measure.

In our applications, we want to go from upper bounds on $b$ and $c$ to one on $g$. Theorem 3.2 is the perfect vehicle for this.

Theorem 3.2. Suppose that $b \geqslant g f(c / 4 g)$, where $f$ is given by (30) and $b, g, c \geqslant 0$. Suppose that $b \leqslant b_{0}$ and $c \leqslant c_{0}$. Then $g \leqslant g_{0}$, where

$$
\begin{aligned}
g_{0} & =\frac{1}{2}\left(c_{0} b_{0}\right)^{1 / 2} \operatorname{coth} x_{0} \\
x_{0}{ }^{2} & =c_{0} / 4 b_{0} .
\end{aligned}
$$


Proof. First note that $g_{0}$ is just chosen so that $g_{0} f\left(c_{0} / 4 g_{0}\right)=b_{0}$. Suppose that $g>g_{0}$. Then $c / 4 g<c_{0} / 4 g_{0}$ and since $f$ is monotone decreasing (see Appendix A),

$$
g f(c / 4 g) \geqslant g_{0} f\left(c_{0} / 4 g_{0}\right)=b_{0} \geqslant b
$$

thereby violating the hypothesis that $b \geqslant g f(c / 4 g)$.

The following will not be needed in our applications but we think it of sufficient interest to mention it.

Theorem 3.3. Under the hypothesis of Theorem 3.2,

$$
b-g \geqslant b_{0}-g_{0}=\frac{1}{2}\left(c_{0} b_{0}\right)^{1 / 2}\left(\frac{1}{x_{0}}-\frac{1}{\tanh x_{0}}\right)
$$

Remark. In the cases of interest, we emphasize that $b-g \leqslant 0$.

Proof. The function $g(f(c / 4 g)-1)$ is seen to be monotone decreasing in both $g$ and $c$ (see Appendix A). Since $g \leqslant g_{0}$ and $c \leqslant c_{0}$,

$$
b-g \geqslant g(f(c / 4 g)-1) \geqslant g_{0}\left(f\left(c_{0} / 4 g_{0}\right)-1\right)=b_{0}-g_{0}
$$

\section{GAUSSIAN DOMINATION-THE QUANTUM CASE}

Our goal in this section is to prove the quantum analog of (10). We will succeed only if all the matrices can be simultaneously chosen to be real. As will be shown later, the antiferromagnet can be accommodated, even though it is essentially complex. This is discussed further in Ref. 50. We will restrict ourselves to nearest neighbor interactions on a simple cubic lattice, but no special commutation properties are required. Thus for each $\alpha$ we choose a copy $\mathscr{H}_{\alpha}$ of the same Hilbert space and copies of $n+1$ basic operators denoted by $S_{\alpha}^{(1)}, \ldots, S_{\alpha}^{(n)}, \tilde{A}_{\alpha}$. To avoid unnecessary technical complications, we suppose $\operatorname{dim} \mathscr{H}_{\alpha}<\infty$, but it is clear that various unbounded operators on infinite-dimensional spaces could be accommodated. The basic Hamiltonian in $\Lambda$ is

$$
\begin{aligned}
H & =\sum_{\alpha \in \Lambda}\left(\tilde{A}_{\alpha}-\sum_{m=1}^{\nu} \sum_{j=1}^{n} S_{\alpha}^{(j)} S_{\alpha+\mathbf{\delta}_{m}}^{(j)}\right) \\
& =\sum_{\alpha \in \Lambda}\left[A_{\alpha}+\frac{1}{2} \sum_{m=1}^{\nu}\left(\mathbf{S}_{\alpha}-\mathbf{S}_{\alpha+\delta_{m}}\right)^{2}\right]
\end{aligned}
$$

where $A_{\alpha}=\tilde{A}_{\alpha}-\nu \mathbf{S}_{\alpha}{ }^{2}$. We define $\widehat{\mathrm{S}}_{\mathbf{p}}$ by (5) and set

$$
b_{\mathbf{p}}^{(j)}=\left(\hat{S}_{\mathbf{p}}^{(j)}, \hat{S}_{-\mathbf{p}}^{(j)}\right)
$$

the Duhamel two-point function. $E_{\mathbf{p}}$ is given by (6). Then we will prove the following result below:

Theorem 4.1. For Hamiltonians of the form (41) in boxes $\Lambda$ of sides $L_{1} \times \cdots \times L_{v}$ with each an $L_{j}$ even integer and such that Theorem 4.2 holds,

$$
b_{\mathbf{p}}^{(j)} \leqslant\left(2 \beta E_{\mathbf{p}}\right)^{-1}
$$


As in the FSS proof of the classical case, we prove (42) on the basis of the following "Gaussian domination" estimate (Theorem 2.1 of Ref. 12). We thank J. Fröhlich for having suggested the following result to us as a conjecture:

Theorem 4.2. Let $H$ be a Hamiltonian of the form (41), in which all the matrices are real. Let $\left\{\mathbf{h}_{i}(\boldsymbol{\alpha}) \mid \boldsymbol{\alpha} \in \Lambda, i=1, \ldots, \nu\right\}$ be $\nu|\Lambda|$ vectors in $\mathbb{R}^{n}$. Let $\partial_{j} \mathbf{h}_{i}(\boldsymbol{\alpha}) \equiv \mathbf{h}_{i}\left(\boldsymbol{\alpha}+\boldsymbol{\delta}_{j}\right)-\mathbf{h}_{i}(\boldsymbol{\alpha})$ and $\sigma(\mathbf{h})=\sum_{\boldsymbol{\alpha}} \mathbf{h}(\boldsymbol{\alpha}) \cdot \mathbf{S}_{\alpha}$. Let $\Lambda$ be $L_{1} \times \cdots \times L_{v}$ with each $L_{i}$ even. Then

$$
\frac{\operatorname{Tr}\left\{\exp \left[-\beta H+\sigma\left(\sum \partial_{i} \mathbf{h}_{i}\right)\right]\right\}}{\operatorname{Tr}(\exp -\beta H)} \leqslant \exp \frac{\|h\|^{2}}{2 \beta}
$$

where $\|h\|^{2}=\sum_{i, \boldsymbol{\alpha}}\left|\mathbf{h}_{i}(\boldsymbol{\alpha})\right|^{2}$.

Proof of Theorem 4.1 Given Theorem 4.2. One can follow FSS; we provide an essentially equivalent proof for the reader's convenience-since the proof below uses no operator theory, it will no doubt be more attractive to some, less attractive to others. Taking $h_{i} \rightarrow \lambda h_{i}$ in (43), subtracting 1 from both sides, dividing by $\lambda^{2}$, and taking $\lambda$ to zero, we find

$$
\left(\sigma \overline{\left(\sum_{i} \partial_{i} \mathbf{h}_{i}\right)}, \sigma\left(\sum_{i} \partial_{i} \mathbf{h}_{i}\right)\right) \leqslant \beta^{-1} \sum_{i, \alpha}\left|\mathbf{h}_{i}(\alpha)\right|^{2}
$$

This equation has just been proven for $h$ real-valued, but by (19) it extends to complex-valued $h$ 's. Fix $\mathbf{p} \neq 0$ and $j$ in $\{1, \ldots, n\}$. Choose now

$$
\left[\mathbf{h}_{i}(\boldsymbol{\alpha})\right]_{k}=\delta_{j k}\left\{\exp \left[i \mathbf{p} \cdot\left(\boldsymbol{\alpha}-\boldsymbol{\delta}_{i}\right)\right]-\exp (i \mathbf{p} \cdot \boldsymbol{\alpha})\right\}|\Lambda|^{-1 / 2}
$$

where the subscript $k$ labels the $n$ components of $\mathbf{h}$. We have

$$
\sum_{i, \alpha}\left|h_{i}(\boldsymbol{\alpha})\right|^{2}=\sum_{i}\left|\exp \left(-i \mathbf{p} \cdot \delta_{i}\right)-1\right|^{2}=2 E_{\mathbf{p}}
$$

while

$$
\left[\sum_{i}\left(\partial_{i} \mathbf{h}_{i}\right)(\boldsymbol{\alpha})\right]_{k}=\delta_{j k}|\Lambda|^{-1 / 2}\left(2 E_{\mathbf{p}}\right) \exp (i \mathbf{p} \cdot \boldsymbol{\alpha})
$$

so that (44) becomes

which is (42).

$$
4 E_{\mathbf{p}}^{2}\left(\hat{\sigma}_{\mathbf{p}}^{(j)}, \hat{\sigma}_{-\mathbf{p}}^{(j)}\right) \leqslant\left(2 E_{\mathbf{p}}\right) \beta^{-1}
$$

We next turn to the proof of Theorem 4.2. In the FSS proof of (10) a critical role was played by the inequality

$$
\begin{aligned}
& \left\{\int \exp \left[-\frac{1}{2}(x-y-a)^{2}\right] d \mu(x) d \nu(y)\right\}^{2} \\
& \quad \leqslant \int \exp \left[-\frac{1}{2}(x-y)^{2}\right] d \mu(x) d \mu(y) \int \exp \left[-\frac{1}{2}(x-y)^{2}\right] d \nu(x) d \nu(y)
\end{aligned}
$$


for any measures $d \mu, d \nu$ on $\mathbb{R}^{n}$ and any $n$-tuple of reals $a$. We begin the proof of Theorem 4.2 with a quantum analog of this fact.

Lemma 4.1. Let $\mathscr{H}_{1}$ be a finite-dimensional vector space and let $\mathscr{H}=\mathscr{H}_{1} \otimes \mathscr{H}_{1}$. If $A, B, \ldots$ are operators on $\mathscr{H}_{1}$, we use the symbols $A, B, \ldots$ for the operators $A \otimes 1, B \otimes 1, \ldots$, and the symbols $\tilde{A}, \widetilde{B}, \ldots$ for $1 \otimes A$, $1 \otimes B, \ldots$. Then for any self-adjoint operators $A, B, C_{1}, \ldots, C_{l}$ with real matrix representations and real numbers $h_{1}, \ldots, h_{l}$

$$
\begin{aligned}
& \left(\operatorname{Tr}\left\{\exp \left[A+\widetilde{B}-\sum_{i=1}^{l}\left(C_{i}-\tilde{C}_{i}-h_{i}\right)^{2}\right]\right\}\right)^{2} \\
& \quad \leqslant \operatorname{Tr}\left\{\exp \left[A+\tilde{A}-\sum_{i=1}^{l}\left(C_{i}-\tilde{C}_{i}\right)^{2}\right]\right\} \operatorname{Tr}\left\{\exp \left[B+\tilde{B}-\sum_{i=1}^{l}\left(C_{i}-\widetilde{C}_{i}\right)^{2}\right]\right\}
\end{aligned}
$$

Proof. Let $\alpha$ denote the quantity being squared on the left-hand side of (45). By the Trotter product formula (see, e.g., Section VIII.8 of Ref. 37), we have that $\alpha=\lim _{n \rightarrow \infty} \alpha_{n}$, where

$$
\alpha_{n}=\operatorname{Tr}\left(\left\{\exp (A / n) \exp (\tilde{B} / n) \exp \left[-\left(C_{1}-\widetilde{C}_{1}-h_{1}\right)^{2} / n\right] \cdots\right\}^{n}\right)
$$

Using the operator identity

$$
\exp \left(-D^{2}\right)=(4 \pi)^{-1 / 2} \int \exp (i k D) \exp \left(-k^{2} / 4\right) d k
$$

we have that

$$
\begin{aligned}
\alpha_{n}= & (4 \pi)^{-n l / 2} \int d^{n l} k \\
& \times \operatorname{Tr}\left\{\exp (A / n) \exp (\tilde{B} / n) \exp \left[i k_{1}\left(C_{1}-\widetilde{C}_{1}\right) / n^{1 / 2}\right] \cdots\right\} \\
& \times \exp \left(-k^{2} / 4\right) \exp \left(-i k_{1} h_{1}+\cdots\right)
\end{aligned}
$$

The operators $A, B$, etc., can be thought of as matrices. We have assumed that they are all real. Then

$$
\begin{aligned}
& \operatorname{Tr}\left\{\exp (A / n) \exp (\tilde{B} / n) \exp \left[i k_{1}\left(C_{1}-\widetilde{C}_{1}\right) / n^{1 / 2}\right] \cdots\right\} \\
& \quad=\operatorname{Tr}\left[\exp (A / n) \exp \left(i k_{1} C_{1} / n^{1 / 2}\right) \cdots\right] \overline{\operatorname{Tr}\left[\exp (B / n) \exp \left(i k_{1} C_{1} / n^{1 / 2}\right) \cdots\right]}
\end{aligned}
$$

where we have used the reality of the matrices $A, B$, etc., to take the complex conjugate without reversing the order of the factors. We have also used the fact that $\widetilde{D}$ and $F$ commute for any two operators $D$ and $F$. Using the 
Schwarz inequality on the $d k$ integration in (46) and then using (47) with $B=A$, we obtain

$$
\begin{aligned}
\left|\alpha_{n}\right|^{2} & \leqslant\left((4 \pi)^{-n l / 2} \int d^{n l} k \operatorname{Tr}\{\exp (A / n) \exp (\tilde{A} / n)\right. \\
& \left.\left.\times \exp \left[i k_{1}\left(C_{1}-\tilde{C}_{1}\right) / n^{1 / 2}\right] \cdots\right\} \exp \left(-k^{2} / 4\right)\right) \\
& \times\left((4 \pi)^{-\pi l / 2} \int d^{n l} k \operatorname{Tr}\{\exp (B / n) \exp (\tilde{B} / n)\right. \\
& \left.\left.\times \exp \left[i k\left(C_{1}-\tilde{C}_{1}\right) / n^{1 / 2}\right] \cdots\right\} \exp \left(-k^{2} / 4\right)\right)
\end{aligned}
$$

Reversing the steps at the start of the proof, we obtain (45).

Remark. In our original announcement ${ }^{(51)}$ we claimed that we could prove a phase transition for the ferromagnet. At the time we believed that Theorem 4.2 held for complex matrices by virtue of the following stratagem: Complex matrices can be made real by doubling the size of the representation. Unfortunately, if one doubles $\mathscr{H}$ then the reflection structure is destroyed. If one doubles $\mathscr{H}_{1}$ then the trace can be changed in a nontrivial way. An illustration of what can happen is provided by the fact that

$$
\operatorname{Tr}\left\{\left(\sum_{i} A_{i} \tilde{A_{i}}\right)^{3}\right\} \geq 0
$$

for real $A_{i}$, whereas for spin matrices,

$$
\operatorname{Tr}(\sigma \cdot \sigma)^{3}<0
$$

Proof of Theorem 4.2. Define

$Z\left(\left\{\mathbf{b}_{i}(\boldsymbol{\alpha})\right\}\right)=\operatorname{Tr}\left[\exp \left(-\sum_{\boldsymbol{\alpha} \in \Lambda}\left\{\beta A_{\alpha}+\frac{\beta}{2} \sum_{m=1}^{\nu}\left[\mathbf{S}_{\boldsymbol{\alpha}}-S_{\alpha+\boldsymbol{\delta}_{m}}+\beta^{-1} \mathbf{h}_{m}(\boldsymbol{\alpha})\right]^{2}\right\}\right)\right]$

Then (43) is easily seen to be equivalent to

$$
Z\left(\left\{\mathbf{h}_{i}(\boldsymbol{\alpha})\right\}\right) \leqslant Z(\{0\})
$$

for all real $\left\{h_{i}(\alpha)\right\}$. Since $Z$ is continuous in the $h$ 's and goes to zero as any $\mathbf{h}_{i}(\boldsymbol{\alpha}) \rightarrow \infty$, it takes its maximum value $Z_{0}$ at some set of $\mathbf{h}$ 's, say $\overline{\mathbf{h}}_{i}(\boldsymbol{\alpha})$. If this maximum value is taken at more than one point, choose a point with the largest number of $h$ 's equal to zero. Thus, we must show $\overrightarrow{\mathbf{h}}_{i}(\alpha)=0$ for all $\alpha, i$. If not, by relabeling we can suppose that $\overline{\mathbf{h}}_{i}(\boldsymbol{\alpha}) \neq 0$ for $i=1$ and $\alpha=$ $\left(L_{1}-1,0, \ldots, 0\right)$. Let $\mathscr{H}_{1}$ be the tensor product of all the $\mathscr{H}_{\gamma}$ such that 
$\gamma=\left(\gamma_{1}, \ldots, \gamma_{v}\right)$ with $0 \leqslant \gamma_{1} \leqslant \frac{1}{2} L_{1}-1$ and $\gamma_{2}, \ldots, \gamma_{\nu}$ arbitrary. Then $\mathscr{H}_{\Lambda}=$ $\mathscr{H}_{1} \otimes \mathscr{H}_{1}$ in such a way that $\tilde{S}_{\gamma}=S_{\gamma}$, where $\tilde{\gamma}_{2}=\gamma_{2}, \ldots, \gamma_{v}=\tilde{\gamma}_{v}, \tilde{\gamma}_{1}=$ $L_{1}-1-\gamma_{1}$. With this representation

$$
Z\left(\left\{\mathbf{h}_{i}(\alpha)\right\}\right)=\operatorname{Tr}\left\{\exp \left[D+\tilde{D}-\sum_{i=1}^{l}\left(C_{i}-\tilde{C}_{i}-y_{i}\right)^{2}\right]\right\}
$$

where $D$ is all "interactions" between $\mathscr{H}_{1}$-spins, the $C_{i}$ represent the spins at sites $\left(0, \gamma_{2}, \ldots, \gamma_{v}\right)$ and $\left(\frac{1}{2} L_{1}-1, \gamma_{2}, \ldots, \gamma_{v}\right)$, and the $y$ 's are the corresponding $h$ 's up to factors of $\beta$.

By Lemma 4.1, we conclude that

$$
Z\left(\left\{\mathbf{h}_{i}(\boldsymbol{\alpha})\right\}\right)^{2} \leqslant Z\left(\left\{\mathbf{h}_{i}^{(1)}(\boldsymbol{\alpha})\right\}\right) Z\left(\left\{\mathbf{h}_{i}^{(2)}(\boldsymbol{\alpha})\right\}\right)
$$

where $\mathbf{h}^{(1)}\left(\right.$ resp. $\left.\mathbf{h}^{(2)}\right)$ is a set of $h$ 's invariant under the $\boldsymbol{\gamma} \rightarrow \tilde{\gamma}$ reflection and equal to the $\overline{\mathbf{h}}$ 's on the $\mathscr{H}_{1}$ (resp. $\tilde{\mathscr{H}}_{1}$ ) spins and zero on the bonds between $\mathscr{H}_{1}$ and $\tilde{\mathscr{H}}_{1}$.

Now, on the one hand either $\left\{\mathbf{h}_{i}^{(1)}(\alpha)\right\}$ or $\left\{\mathbf{h}_{i}^{(2)}(\alpha)\right\}$ must contain strictly more zero elements than $\left\{\overline{\mathbf{h}}_{i}(\alpha)\right\}$ and on the other hand since $Z\left(\left\{\overline{\mathbf{h}}_{i}(\boldsymbol{\alpha})\right\}\right)=Z_{0}$ and $Z\left(\left\{\mathbf{h}_{i}(\alpha)\right\}\right) \leqslant Z_{0}$, we must have $Z\left(\left\{\mathbf{h}_{i}(\alpha)\right\}\right)=Z_{0}$. This contradicts the fact that the $\bar{h}$ has a maximal number of zeros, so it must be that all $\bar{h}$ 's are zero.

We conclude this section with a few remarks on the restrictions we have placed on the interaction. It is our belief that the basic bound (48), suitably generalized to allow $h$ 's on each bond, holds for any ferromagnetic interaction, but we are in the unhappy situation of not being able to prove it (or phase transitions) even for face-centered or body-centered cubic lattices. Our proof is restricted to lattices with the following property: The perpendicular bisector of any bond contains no sites and the lattice is reflection symmetric about that bisector. For example, the two-dimensional honeycomb (hexagonal) lattice can be handled.

\section{PHASE TRANSITIONS: THE FERROMAGNETIC CASE}

In this section we want to put the results of Sections 3 and 4 together with explicit calculations of the double commutator to prove that phase transitions occur in the spin $1 / 2 x-y$ model, where the matrices can be chosen simultaneously real, namely

$$
S_{x}=1 / 2\left(\begin{array}{ll}
0 & 1 \\
1 & 0
\end{array}\right), \quad S_{y}=1 / 2\left(\begin{array}{rr}
1 & 0 \\
0 & -1
\end{array}\right)
$$

This is Example 4 below. We also present the consequences, in terms of phase transitions, that would follow if Theorem 4.2 held for the ferromagnet; these are contained in Examples 1, 2, and 3. 
We first rephrase the abstract result, Theorem 3.2. Suppose that we have a lattice system on $\mathbb{Z}^{v}$ (with no a priori restriction on the kind of interactions) with operators $S_{\alpha}^{(j)}$ at each site. Define $\hat{S}_{p}^{(j)}$ in the usual way and

$$
\begin{array}{rlrl}
d^{(j)} & =\left\langle S_{\alpha}^{(j)} S_{\alpha}^{(j)}\right\rangle ; & d & =\sum_{j} d^{(j)} \\
b_{\mathbf{p}}^{(j)} & =\left(\hat{S}_{\mathbf{p}}^{(j)}, \hat{S}_{-\mathbf{p}}^{(j)}\right) ; & b_{\mathbf{p}}=\sum_{j} b_{\mathbf{p}}^{(j)} \\
g_{\mathbf{p}}^{(j)}=\left\langle\hat{S}_{\mathbf{p}}^{(j)} \hat{S}_{-\mathbf{p}}^{(j)}\right\rangle ; & g_{\mathbf{p}}=\sum_{j} g_{\mathbf{p}}^{(j)} \\
c_{\mathbf{p}}^{(j)}=\left\langle\left[\hat{S}_{\mathbf{p}}^{(j)},\left[\beta H, \hat{S}_{-\mathbf{p}}^{(j)}\right]\right]\right\rangle \geqslant 0 ; & c_{\mathbf{p}}=\sum_{j} c_{\mathbf{p}}^{(j)}
\end{array}
$$

(Note that $S_{\mathbf{p}}^{(j)}$ and $\hat{S}_{\mathbf{q}}^{(j)}$ commute for any $\mathbf{p}$ and $\mathbf{q}$.)

From the sum rule (Plancherel)

$$
\frac{1}{|\Lambda|} \sum_{\mathbf{p} \in \Lambda^{*}} g_{\mathbf{p}}^{(j)}=d^{(j)}
$$

and Theorem 3.2, one immediately concludes that:

Theorem 5.1. Suppose that there exist fixed measurable functions $B_{\mathbf{p}}^{(j)}, C_{\mathbf{p}}^{(j)}$ of $\mathbf{p}$ and a function $D^{(j)}(\beta)$ such that for every finite system $L_{1} \cdots L_{\nu}$ with $L_{i}$ even, one has the bounds
(a) $d^{(j)} \geqslant D^{(j)}(\beta)$ and $\lim _{\beta \rightarrow \infty} D^{(j)}(\beta) \geqslant D_{\infty}^{(j)}$.
(b) $b_{\mathbf{p}}^{(j)} \leqslant \beta^{-1} B_{\mathbf{p}}^{(j)} ; B_{\mathbf{p}}<\infty$ for $p \neq 0$.
(c) $c_{\mathbf{p}}^{(j)} \leqslant \beta C_{\mathbf{p}}^{(j)}$.

Further assume that

$$
\lim _{|\Lambda| \rightarrow \infty}|\Lambda|^{-1} \sum_{\substack{\mathbf{p} \neq 0 \\ \mathbf{p} \in \Lambda^{*}}} B_{\mathbf{p}}=\int B_{\mathbf{p}} d^{v} p /(2 \pi)^{v}
$$

Then there is long-range order at some finite $\beta$ whenever (49) and (50) hold for some $j$ :

$$
\begin{gathered}
D_{\infty}^{(j)}>(2 \pi)^{-v} \int_{\left|p_{\mathfrak{i}}\right| \leqslant \pi} \frac{1}{2}\left(B_{\mathbf{p}}^{(j)} C_{\mathbf{p}}^{(j)}\right)^{1 / 2} d^{v} p \\
\int_{\left|p_{1}\right| \leqslant \pi} B_{\mathbf{p}}^{(j)} d^{v} p<\infty ; \quad D_{\infty}^{(j)}<\infty
\end{gathered}
$$

There is long-range order for any $\beta$ such that

$$
D^{(j)}(\beta)>(2 \pi)^{-v} \int_{\left|p_{i}\right| \leqslant \pi} \frac{1}{2}\left(B_{\mathbf{p}}^{(j)} C_{\mathbf{p}}^{(j)}\right)^{1 / 2} \operatorname{coth}\left[\frac{1}{2} \beta\left(C_{\mathbf{p}}^{(j)} / B_{\mathbf{p}}^{(j)}\right)^{1 / 2}\right] d^{v} p
$$


In particular, if $D^{(j)}(\beta)$ is monotone nondecreasing in $\beta$ and (49) and (50) hold, then there is a long-range order for $\beta>\widetilde{\beta}_{c}$, where $\widetilde{\beta}_{c}$ is the unique solution of

$$
D^{(j)}\left(\tilde{\beta}_{c}\right)=(2 \pi)^{-v} \int_{\left|p_{i}\right| \leqslant \pi} \frac{1}{2}\left(B_{\mathbf{p}}^{(j)} C_{\mathbf{p}}^{(j)}\right)^{1 / 2} \operatorname{coth}\left[\frac{1}{2} \tilde{\beta}_{c}\left(C_{\mathbf{p}}^{(j)} / B_{\mathbf{p}}^{(j)}\right)^{1 / 2}\right] d^{v} p
$$

Proof. Theorem 3.2 and the stated sum rule yield (4) whenever (51) holds. When (49) and (50) hold, (51) must hold for $\beta$ large. To prove this, (50) is used together with the bound (see Appendix A)

$$
\operatorname{coth} x \leqslant x^{-1}+1, \quad x \geqslant 0
$$

and the dominated convergence theorem to obtain (49) as the $\beta \rightarrow \infty$ limit of (51). Equation (52) has a unique solution since the right side of (51) decreases strictly monotonically from $\infty$ to

$$
(2 \pi)^{-v} \int_{\left|p_{1}\right| \leqslant \pi} \frac{1}{2}\left(B_{\mathbf{p}}^{(j)} C_{\mathbf{p}}^{(j)}\right)^{1 / 2} d^{v} p \quad \text { as } \quad \beta \rightarrow \infty
$$

Remark. If all superscripts $j$ are replaced by dot products (i.e., $\sum_{j=1}^{3}$ in the usual Heisenberg case), the result still holds.

Example 1. Usual [isotropic, $O(3)$ ] Heisenberg model of spin $S$. This is the model described in Section 1. For nearest neighbor simple cubic coupling, we consider the consequence of supposing that we may take $B_{\mathrm{p}}=3 /\left(2 E_{\mathrm{p}}\right)$. By a simple calculation (see below), we can take $C_{\mathrm{p}}=4 S E_{\mathrm{p}}$. Finally, $D(\beta)=S(S+1)$ can obviously be taken. Thus (50) holds as long as $\nu \geqslant 3$ and (49) becomes

$$
S(S+1)>S \sqrt{\frac{3}{2}}
$$

which holds for $S=1 / 2,1,3 / 2$, etc. In order to solve (52), one needs the function (in $v=3$ dimensions)

$$
H_{3}(x)=(2 \pi)^{-3} \int_{\left|p_{1}\right| \leqslant \pi} \operatorname{coth}\left(x E_{\mathrm{p}}\right) d^{3} p
$$

which is tabulated in Table I and graphed in Fig. 2 (we owe these to J. F. Barnes). The solutions of

$$
S(S+1)=S \sqrt{\frac{3}{2}} H_{3}\left(\beta S \sqrt{\frac{2}{3}}\right)
$$

are shown in Table II (also due to J. F. Barnes).

For use below, we do the double commutator calculation in the general case. Let $H$ be given by (41) and define

$$
i Q_{\alpha}^{i j}=\left[S_{\alpha}^{(i)}, S_{\alpha}^{(j)}\right], \quad i P_{\alpha}^{k ; i j}=\left[S_{\alpha}^{(k)}, Q_{\alpha}^{i j}\right], \quad R_{\alpha}^{i j}=\left[S_{\alpha}^{(i)},\left[A_{\alpha}, S_{\alpha}^{(j)}\right]\right]
$$




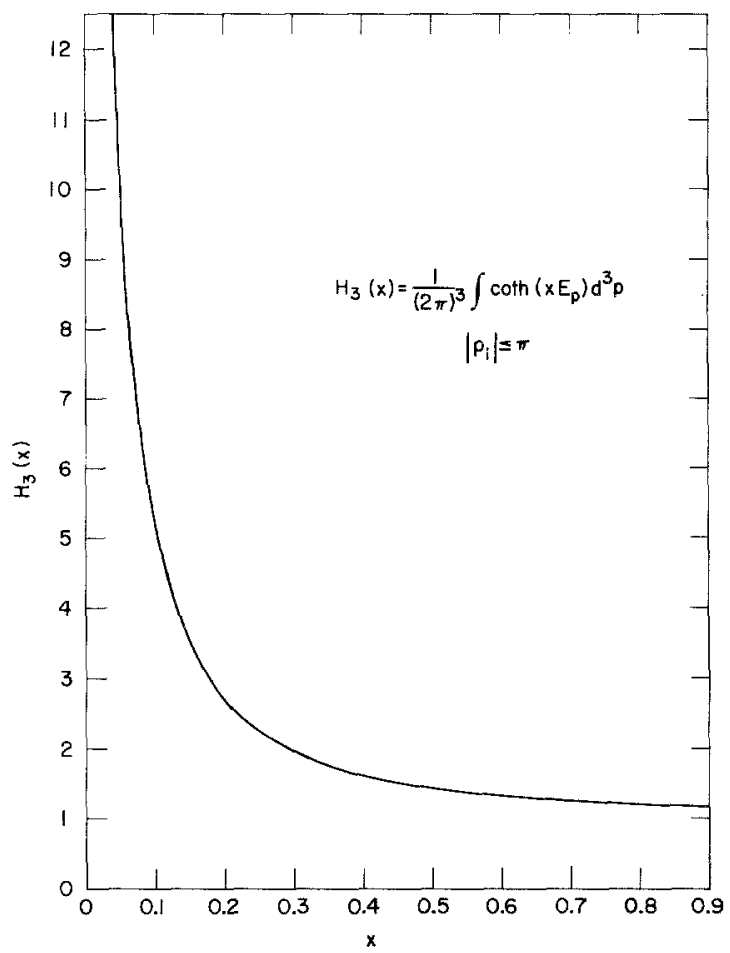

Fig. 2. Graph of the integral appearing in $(52 H)$ for three dimensions. The large- $x$ asymptotic form of $H_{v}(x)$ in $v$ dimensions is given by $H_{v}(x) \sim 1+B_{v} x^{-v / 2}$ and $B_{v}=2^{-v+1} \pi^{-v / 2} \zeta(v / 2)$, with $\zeta$ being the Riemann zeta function. $B_{3}=0.117$. (Values computed by J. F. Barnes.)

Then, by a direct calculation,

$\left[\hat{S}_{-\mathbf{p}}^{(i)},\left[H, \hat{S}_{\mathbf{p}}^{(i)}\right]\right]$

$=-\frac{1}{\Lambda} \sum_{\alpha}\left\{R_{\alpha}^{i i}+\sum_{m=1}^{\nu} \sum_{j=1}^{n}\left[P_{\alpha}^{i i j} S_{\alpha+\delta_{m}}^{(j)}+S_{\alpha}^{(j)} P_{\alpha+\delta_{m}}^{i i j}+2 \cos \left(\mathbf{p} \cdot \delta_{m}\right) Q_{\alpha}^{i j} Q_{\alpha+\delta_{m}}^{i j}\right]\right\}$

In the case of Example $1, R=0, Q^{i j}=\epsilon^{i j k} S^{(k)}, \sum_{i} P^{i j j}=-2 S^{(j)}$, from which one concludes that

$$
\sum_{i}\left[\hat{S}_{-\mathbf{p}}^{(i)},\left[H, \hat{S}_{\mathbf{p}}^{(i)}\right]\right]=\frac{1}{|\Lambda|} \sum_{\alpha \in \Lambda} \sum_{m=1}^{v} 4\left(1-\cos \mathbf{p} \cdot \delta_{m}\right) \mathbf{S}_{\alpha} \mathbf{S}_{\alpha+\boldsymbol{\delta}_{m}}
$$

In particular, as an operator,

$$
\sum_{i}\left[\hat{S}_{-\mathbf{p}}^{(i)},\left[H, \hat{S}_{\mathbf{p}}^{(i)}\right]\right] \leqslant 4 E_{\mathbf{p}} S^{2}
$$


Table I. A Table of Values of the Function Shown in Fig. 2

\begin{tabular}{rrrr}
\hline$y$ & \multicolumn{1}{c}{$H_{3}(y)$} & $y$ & $H_{3}(y)$ \\
\hline 0.01 & 50.5562 & 0.75 & 1.2182 \\
0.05 & 10.1591 & 0.80 & 1.1956 \\
0.10 & 5.1537 & 0.85 & 1.1766 \\
0.15 & 3.5168 & 0.90 & 1.1604 \\
0.20 & 2.7206 & 0.95 & 1.1465 \\
0.25 & 2.2591 & 1.00 & 1.1345 \\
0.30 & 1.9637 & 1.50 & 1.0693 \\
0.35 & 1.7621 & 2.00 & 1.0439 \\
0.40 & 1.6180 & 2.50 & 1.0310 \\
0.45 & 1.5114 & 3.00 & 1.0234 \\
0.50 & 1.4305 & 4.00 & 1.0150 \\
0.55 & 1.3676 & 5.00 & 1.0107 \\
0.60 & 1.3178 & 6.00 & 1.0081 \\
0.65 & 1.2778 & 8.00 & 1.0052 \\
0.70 & 1.2451 & 10.00 & 1.0037 \\
\hline
\end{tabular}

since $\mathbf{S}_{\boldsymbol{\alpha}} \cdot \mathbf{S}_{\boldsymbol{\beta}} \leqslant S^{2}$. In any state such that $\left\langle\mathbf{S}_{\alpha} \cdot \mathbf{S}_{\boldsymbol{\alpha}+\boldsymbol{\boldsymbol { \delta }}_{m}}\right\rangle$ is independent of $\alpha$ and $m$,

$$
\left\langle\sum_{i}\left[\hat{S}_{-\mathbf{p}}^{(i)},\left[H, \hat{S}_{\mathbf{p}}^{(i)}\right]\right]\right\rangle=-4 E_{\mathbf{p}}|\Lambda|^{-1} \nu^{-1}\langle H\rangle
$$

The fact that the $P \cdot S$ and $Q \cdot Q$ terms combine so nicely is coincidental (see Examples 3 and 4 below).

Example 2. $O(n)$ Heisenberg model. Instead of three spin operators $S_{a}^{(i)}(i=1,2,3)$ obeying the $O(3)$ commutation relations, we have $n(n-1) / 2$ spin operators obeying the $O(n)$ commutation relations with the same irreducible representation $R$ at each point.

The commutation relations have each spin operator commuting with $(n-2)(n-3) / 2$ other spin operators. The other $2(n-2)$ spin operators

Table II. Lower Bounds on the Critical $\beta$ in Three Dimensions [Solutions of $(52 H)$ ]

\begin{tabular}{cccc}
\hline$S$ & $\beta_{c}(S) S(S+1)$ & $S$ & $\beta_{c}(S) S(S+1)$ \\
\hline$\frac{1}{2}$ & 1.354 & $\frac{7}{2}$ & 0.788 \\
1 & 0.965 & 4 & 0.783 \\
$\frac{3}{2}$ & 0.872 & $\frac{9}{2}$ & 0.778 \\
2 & 0.832 & 5 & 0.775 \\
$\frac{5}{2}$ & 0.810 & $\infty$ & 0.758 \\
3 & 0.797 & & \\
\hline
\end{tabular}


break into $n-2$ pairs, which, along with the given spin, form $n-2$ copies of $O(3)$. Using these facts, one quickly computes as above that one can take as a bound on the double commutator

$$
C_{\mathbf{p}}=4(n-2) \tilde{K} E_{\mathbf{p}}
$$

where $\widetilde{K}$, depending on the representation $R$, is the maximum eigenvalue of $\mathbf{S}_{\alpha} \cdot \mathbf{S}_{\boldsymbol{\beta}}($ for $\alpha \neq \beta)$. The putative Duhamel function bound $B_{\mathbf{p}}$ is $n(n-1)\left[4 E_{\mathbf{p}}\right]^{-1}$. Finally, let $K$ be the value, depending on $R$, of $\sum\left(S^{(i)}\right)^{2}$. Then the condition for a phase transition in $\nu \geqslant 3$ dimensions would be

$$
K>\widetilde{K}^{1 / 2}\left[\frac{1}{4} n(n-1)(n-2)\right]^{1 / 2}
$$

if one could extend Theorem 4.2. We consider the following examples.

(i) Identity representation. The "natural" representation of $O(n)$ on $\mathbb{C}^{n}$ by real rotations (spin 1 for $n=3$ ) has $K=n-1$ [each $S^{(i)}$ obeys $\operatorname{Tr}\left(S^{(i)}\right)^{2}$ $=2$ ]. Moreover, $\widetilde{K}=1$-because the theory of weights ${ }^{(42)}$ implies that the maximum value of $\mathbf{S}_{\alpha}+\mathbf{S}_{\boldsymbol{\beta}}$ occurs in a space including a vector of the form $u \otimes v$, so that the maximum value is of the form $\left(u, S_{\alpha} u\right) \cdot\left(v, S_{\beta} v\right)$ and since $\operatorname{Tr}\left(\mathbf{S}_{\boldsymbol{\alpha}} \cdot \mathbf{a}\right)=0, \operatorname{Tr}\left[\left(S_{\alpha} \cdot \mathbf{a}\right)^{2}\right]=2|\mathbf{a}|^{2}$ for any $\frac{1}{2} n(n-1)$-vector $\mathbf{a},\left(u, \mathbf{S}_{\alpha} u\right)$ is always a vector of norm less than or equal to 1 . The condition (56) holds for $n=3,4,5$ but not for $n \geqslant 6$.

(ii) $n=4$. It is well known ${ }^{(42)}$ that as a Lie algebra $O(4)=O(3) \oplus O(3)$. Thus representations $R$ are labeled by pairs $(j, k)$ of half-integers. By direct computation, $K=2[j(j+1)+k(k+1)]$ and $\tilde{K}=2\left[j^{2}+k^{2}\right]$. One can see that (56) holds for all values of $(j, k)$ except for the values $(j, k)=(0,0)$ and $(0,1 / 2)$.

The moral of these computations is the following: When putting together the bounds of Sections 3 and 4, detailed estimates on algebraic properties are needed, which one can sometimes prove and sometimes not. It is fortunate that for the usual ferromagnetic $O(3)$ model one can always verify the algebraic conditions. We should emphasize that we believe phase transitions occur in any quantum system of the type discussed in Section 4 (with not all the $S_{\alpha}$ constant); it is our method that has limitations.

Example 3. Anisotropic Heisenberg model. The anisotropic model is like the isotropic model of Section 1, except that

$$
H=-\sum_{\alpha \in \Lambda} \sum_{m=1}^{v}\left(\sum_{i=1}^{3} J_{i} S_{\alpha}^{(i)} S_{\alpha+\boldsymbol{\delta}_{m}}^{(i)}\right)
$$

We want to consider the prolate case $J_{3}=1, J_{1}=J_{2}$ smaller than 1 (but positive). This is a generalization of the case treated by Ginibre ${ }^{(13)}$ and Robinson $^{(39)}$ and if $\nu \geqslant 3$, we would, modulo the proof of Theorem 4.2, recover their results. In fact, our method is ideal for discussing quantum models of the sort that are small perturbations of classical models. For, if we 
try to prove (49) with $S^{(j)}$ being the "classical" spin about which one is perturbing, in this case $j=3$, then the left side of (49) will have a nonzero limit as the perturbation parameter goes to zero, and the right side will go to zero. In the case at hand, we first argue that

$$
\lim _{\beta \rightarrow \infty} D^{(3)}(\beta)=S^{2}
$$

and that (in infinite volume)

$$
\lim _{\beta \rightarrow \infty}\left\langle S_{a}^{(i)} S_{a+\delta_{m}}^{(i)}\right\rangle=S^{2} \delta_{i 3}
$$

Clearly, (57) follows from (58) and the Schwarz inequality

$$
\left\langle S_{\alpha}^{(3)} S_{\alpha+\delta_{m}}^{(3)}\right\rangle \leqslant\left\langle S_{\alpha}^{(3)} S_{\alpha}^{(3)}\right\rangle \leqslant S^{2}
$$

in translation-invariant states. To prove (58), note that $S_{\alpha} \cdot \mathrm{S}_{\beta} \leqslant S^{2}$ and $S_{\alpha}^{(3)} S_{\beta}^{(3)} \leqslant S^{2}$ imply that $\langle H\rangle /|\Lambda| \leqslant \nu S^{2}$, while the bound $Z_{\Lambda} \geqslant \exp \left(\beta \nu S^{2}\right)$ (from the all-spin-up state) plus a standard convexity argument implies that

$$
\lim _{\beta \rightarrow \infty} \sum_{m=1}^{\nu}\left[J_{1}\left\langle\mathbf{S}_{\alpha} \cdot \mathbf{S}_{\alpha+\delta_{m}}\right\rangle+\left(1-J_{1}\right)\left\langle S_{\alpha}^{(3)} S_{\alpha+\boldsymbol{\delta}_{m}}^{(3)}\right\rangle\right]=\nu S^{2}
$$

From this it follows that $\left\langle S_{\alpha}^{(3)} S_{\alpha+\delta_{m}}^{(3)}\right\rangle$ and $\left\langle S_{\alpha} \cdot S_{\alpha+\delta_{m}}\right\rangle$ both go to $S^{2}$, which implies (58).

Now, suppose we can take $B_{\mathbf{p}}^{(3)}=1 / 2 E_{\mathbf{p}}$. Using (58) and (53),

$$
\lim _{\beta \rightarrow \infty}\left\langle\left[\hat{S}_{-\mathbf{p}}^{(3)},\left[H, \hat{S}_{\mathbf{p}}^{(3)}\right]\right]\right\rangle=0
$$

Thus, a simple modification of (49) (allowing $C_{\mathbf{p}}$ to be $\beta$ dependent) clearly holds. We conclude that in $\nu=3$ dimensions the prolate anisotropic model $\left(J_{3}>J_{1}=J_{2}\right)$ would have a phase transition for arbitrary spin if Theorem 4.2 extends to this case.

Example 4. The $x-y$ model. This is the highly oblate anisotropic case $\left(J_{1}=J_{2}=1, J_{3}=0\right)$. We will verify (49) in this case without the superscripts, i.e., sum over $j=1$ and 2 . Clearly $D(\beta)=\frac{1}{2}$ can be taken for all $\beta$ and $B_{\mathbf{p}}=1 / E_{\mathbf{p}}$. Thus, if $\nu \geqslant 3,(50)$ holds. By the Schwarz inequality, (49) then holds if $\nu \geqslant 3$ and

$$
\left[\int C_{\mathbf{p}} d^{v} p(2 \pi)^{v}\right] G_{v}(0)<1
$$

where $G_{v}(0)=(2 \pi)^{-v} \int E_{\mathbf{p}}^{-1} d^{v} p$. By (53),

$$
\begin{aligned}
(2 \pi)^{-v} \int C_{\mathbf{p}} d^{v} p & =\sum_{i=1}^{2} \sum_{m=1}^{v} \sum_{j=1}^{2}\left\langle P_{\alpha}^{i i j} S_{\alpha+\delta_{m}}^{(j)}+S_{\alpha}^{(j)} P_{\alpha+\delta_{m}}^{i i j}\right\rangle \\
& =\sum_{m=1}^{v} \sum_{\nu= \pm 1}\left\langle S_{\alpha}^{(x)} S_{\alpha+\gamma \delta_{m}}^{(x)}+S_{\alpha}^{(j)} S_{\alpha+\gamma \hat{\boldsymbol{\delta}}_{m}}^{(j)}\right\rangle \\
& \leqslant \frac{1}{2}[\nu(\nu+1)]^{1 / 2}
\end{aligned}
$$


where the last inequality follows from a bound in Appendix $\mathrm{C}$ on the groundstate energy in the $x-y$ model. Thus (59) follows from

$$
\frac{1}{2}\left[\nu G_{\nu}(0)\right]\left(1+\nu^{-1}\right)^{1 / 2}<1
$$

We will prove in Section 7 that $\nu G_{\nu}(0)$ is monotone decreasing in $\nu$, so the largest value of (60) with $\nu \geqslant 3$ occurs when $\nu=3$, where (60) is seen to be true using Watson's value ${ }^{(45)}$ of $G_{3}(0)=0.505 \cdots$. We conclude that (49) holds in the $x-y$ model in any dimension $\nu \geqslant 3$.

We have thus verified (49) and therefore (4) for the $x-y$ model. By what we have done so far this does not imply a phase transition. Theorem 1.2 is not applicable because $\sum_{\alpha \in \Lambda} S_{\alpha}^{(1)}$ does not commute with $H$. Fortunately, the following alternative argument leading to a phase transition is available. Instead of proving that

$$
\lim _{\Lambda \rightarrow \infty}\left\langle\left(|\Lambda|-1 \sum_{\alpha \in \Lambda} \mathbf{S}_{\alpha}\right)^{2}\right\rangle \neq 0
$$

we will in essence prove that

$$
\lim _{\Lambda^{\prime} \rightarrow \infty}\left(\lim _{\Lambda \rightarrow \infty}\left\langle\left(\left|\Lambda^{\prime}\right|-1 \sum_{\alpha \in \Lambda^{\prime}} S_{\alpha}\right)^{2}\right\rangle\right) \neq 0
$$

which directly implies nonclustering of the infinite-volume state and thus the existence of multiple phases. To prove this last result requires us to transfer our bound on $g_{\mathrm{p}}$ to infinite volume, which we will do by some standard manipulations of smearing spins with nice $x$-space functions.

Let $f$ be a function of compact support on $Z^{\nu}$. Define $g^{A}(f)$ by

$$
\begin{aligned}
g^{\Lambda}(f) & =\left\langle\left|\sum_{\alpha \in \Lambda} S_{\alpha}^{(1)} f(\boldsymbol{\alpha})\right|^{2}\right\rangle_{\Lambda} \\
& =\sum_{\alpha, \beta \in \Lambda} \overline{f(\boldsymbol{\alpha})} f(\boldsymbol{\beta})\left\langle S_{\alpha}^{(1)} S_{\boldsymbol{\beta}}^{(1)}\right\rangle_{\Lambda} \\
& =\frac{1}{|\Lambda|} \sum_{\mathbf{p} \in \Lambda^{*}}\left|\hat{f}^{(\Lambda)}(\mathbf{p})\right|^{2} g_{\mathbf{p}}^{(\Lambda)}
\end{aligned}
$$

where $g_{\mathbf{p}}^{(\Lambda)}=\left\langle\hat{S}_{\mathbf{p}}^{(1)} \hat{S}_{-\mathbf{p}}^{(1)}\right\rangle_{\Lambda}$ and

$$
f^{(\Lambda)}(\mathbf{p})=\sum_{\alpha \in \Lambda} f(\alpha) \exp (+i \mathbf{p} \cdot \boldsymbol{\alpha})
$$

Now, for $\mathbf{p} \neq 0$

$$
g_{\mathbf{p}}^{(\Lambda)} \leqslant G_{\mathbf{p}}=\frac{1}{2}\left(B_{\mathbf{p}}^{(1)} C_{\mathbf{p}}^{(1)}\right)^{1 / 2} \operatorname{coth}\left[\frac{1}{2} \beta\left(C_{\mathbf{p}}^{(1)} / B_{\mathbf{p}}^{(1)}\right)^{1 / 2}\right]
$$

so that, picking a subsequence of $\Lambda$ for which the state $\langle\cdots\rangle$ converges,

$$
\lim _{\Lambda \rightarrow \infty} g^{\Lambda}(f) \leqslant(2 \pi)^{-v} \int_{\left|p_{i}\right| \leqslant \pi}|\hat{f}(\mathbf{p})|^{2} G_{\mathbf{p}} d^{v} p
$$


whenever $\hat{f}(\mathbf{p}=0)=0$. [The sum converges to the integral because $f$ has compact support, so $|\hat{f}(\mathbf{p})| \leqslant C|\mathbf{p}|$ if $\hat{f}(0)=0$.] Let $F$ be defined by

$$
F(\alpha-\beta)=\left\langle S_{a}^{(1)} S_{\beta}^{(1)}\right\rangle_{\infty}
$$

and let $\hat{F}$ be the measure given by the Fourier transform of $F$, i.e., formally

$$
\hat{F}(\mathbf{p})=\sum_{\alpha}[\exp (i \mathbf{p} \cdot \boldsymbol{\alpha})] F(\boldsymbol{\alpha})
$$

Thus, since the sum in (61) is finite

$$
\begin{aligned}
\int|\hat{f}(\mathbf{p})|^{2} \hat{F}(\mathbf{p}) d^{v} p & =\sum_{\boldsymbol{\alpha}, \boldsymbol{\beta}} \overline{f(\boldsymbol{\alpha})} f(\boldsymbol{\beta}) F(\boldsymbol{\alpha}-\boldsymbol{\beta}) \\
& =\lim _{\Lambda \rightarrow \infty} g^{\Lambda}(f) \leqslant \int|\hat{f}(\mathbf{p})|^{2} G_{\mathbf{p}} d^{v} p
\end{aligned}
$$

whenever $\hat{f}(0)=0$. It follows by letting $\hat{f}$ approach a $\delta$-function at $\mathbf{p} \neq 0$ that

$$
\hat{F}(\mathbf{p})=C \delta(\mathbf{p})+\text { absolutely continuous in } p
$$

with $|\hat{F}(\mathbf{p})| \leqslant G_{\mathbf{p}}$ for $\mathbf{p} \neq 0$. By the Plancherel relation,

$$
\lim _{\beta \rightarrow \infty} C \geqslant D_{\infty}^{(1)}-(2 \pi)^{-v} \int_{\left|p_{i}\right| \leq \pi} \frac{1}{2}\left(B_{\mathbf{p}}^{(1)} C_{\mathbf{p}}^{(1)}\right)^{1 / 2} d^{v} p
$$

so that (49) implies that $\langle\cdots\rangle_{\infty}$ does not cluster and there is a multiplicity of phases. ${ }^{(41)}$

We summarize Example 4 in a theorem:

Theorem 5.2. The spin $1 / 2 x-y$ model with nearest neighbor interactions on a simple cubic lattice in $\nu \geqslant 3$ dimensions possesses a phase transition at sufficiently low temperatures.

As a final remark, we note that even if we could complete the proof of the existence of the phase transition in the isotropic model, we presumably do not get the correct value for the spontaneous magnetization as $T \rightarrow 0$. For, we would obtain (from Theorem 1.3)

$$
\lim _{\overline{T \rightarrow 0}} m\left(S^{(3)}\right)^{2} \geqslant S\left(S+1-\sqrt{\frac{3}{2}}\right)
$$

while the correct value as $T \rightarrow 0$ is presumably $S^{2}$. 


\section{PHASE TRANSITIONS: THE ANTIFERROMAGNETIC CASE}

In the classical case, once one proves phase transitions for simple cubic, nearest neighbor ferromagnets, one automatically has them also for the antiferromagnets since the symmetry $\mathbf{S}_{\alpha} \rightarrow(-1)^{|\alpha|} \mathbf{S}_{\alpha}\left(|\alpha|=\sum_{i=1}^{v} \alpha_{i}\right)$ takes one Hamiltonian into the other. A similar argument works for certain quantum systems, e.g., the $x-y$ model, since there is a unitary operator that takes $\mathbf{S}_{a}^{(i)} \rightarrow(-1)^{|\alpha|} \mathbf{S}_{a}^{(i)}$ for $i=1,2$ (namely rotation by $\pi$ about the $z$ axis of those $\alpha$ with $|\alpha|$ odd). However, for the isotropic quantum Heisenberg model no such symmetry exists: Even for two spins, the smallest eigenvalue of $-S_{\alpha} \cdot S_{\beta}(\alpha \neq \beta)$ (which is $-S^{2}$ ) is different from the smallest eigenvalue of $S_{\alpha} \cdot S_{\beta}(\alpha \neq \beta)$ [which is $-S(S+1)$ ]. In this section we shall prove that the antiferromagnet has phase transitions if either $S$ or $v$ is not very small.

We still use Theorem 5.1 (with some obvious modifications). To complete the proof, we need a modified version of the argument of Example 4 of Section 5, since the staggered magnetization does not commute with $H$. We have already computed $\sum_{i}\left[S_{\alpha}^{(i)},\left[S_{\alpha}^{(i)}, H\right]\right]$ since the ferromagnetic and antiferromagnetic Hamiltonians differ only by a sign. Knowing this, we will turn below to estimating $C_{\mathrm{p}}$. Obviously we can take $D=S(S+1)$. Thus our main problem will be to estimate $B_{\mathrm{p}}$, to which we turn first. Theorem 4.2 , suitably modified, can be extended to the case of ferromagnetically coupled real matrices and antiferromagnetically coupled imaginary matrices. By a local rotation, the quantum antiferromagnet can be brought into this form.

Theorem 6.1. Let $H$ be the Hamiltonian of the simple cubic Heisenberg antiferromagnet with nearest neighbor interactions. Then

$$
\sum_{j=1}^{3} b_{\mathbf{p}}^{(j)} \leqslant 3 / 2 E_{\mathbf{p}}{ }^{\prime}
$$

where

$$
E_{\mathbf{p}}^{\prime}=\nu+\sum_{i=1}^{v} \cos p_{i}
$$

The proof is similar to that of Theorem 4.1, so we only sketch the details, emphasizing the differences. Instead of Lemma 4.1, we need the following result:

Lemma 6.1. Let $\mathscr{H}_{1}$ be a finite-dimensional vector space with a distinguished complex conjugation and let $\mathscr{H}=\mathscr{H}_{1} \otimes \mathscr{H}_{1}$. If $A$ is an operator on $\mathscr{H}_{1}$, we use $A$ for $A \otimes 1$ and $\tilde{A}$ for $1 \otimes A$. If $A, B, C_{1}, \ldots, C_{l}$ are real self-adjoint operators and $D_{1}, \ldots, D_{k}$ are imaginary self-adjoint operators and $h_{1}, \ldots, h_{l}$ are real numbers, then

$$
\{\operatorname{Tr}[\exp (X)]\}^{2} \leqslant \operatorname{Tr}[\exp (Y)] \operatorname{Tr}[\exp (Z)]
$$


where

$$
\begin{aligned}
& X=A+\widetilde{B}-\sum_{i=1}\left(C_{i}-\widetilde{C}_{i}-h_{i}\right)^{2}+\sum_{j=1}^{k}\left(D_{j}-\widetilde{D}_{j}\right)^{2} \\
& Y=A+\tilde{A}-\sum_{i=1}^{l}\left(C_{i}-\widetilde{C}_{i}\right)^{2}+\sum_{j=1}^{k}\left(D_{j}-\widetilde{D}_{j}\right)^{2} \\
& Z=B+\widetilde{B}-\sum_{i=1}^{l}\left(C_{i}-\widetilde{C}_{i}\right)^{2}+\sum_{j=1}^{k}\left(D_{j}-\tilde{D}_{j}\right)^{2}
\end{aligned}
$$

Proof. This follows the proof of Lemma 4.1, except that we use

$$
\exp \left(F^{2}\right)=(4 \pi)^{-1 / 2} \int \exp \left(-\frac{1}{4} k^{2}+k F\right) d k
$$

to "linearize" the $\exp (D-\tilde{D})^{2}$ factors.

Proof of Theorem 6.1. Let $\mathbf{p}^{\prime}$ be given by $p_{i}{ }^{\prime}=\pi-p_{i}$ so that $E_{\mathbf{p}}{ }^{\prime}=E_{\mathbf{p}^{\prime}}$. Define $\mathrm{S}_{\alpha}{ }^{\prime}$ by

$$
\begin{aligned}
& \left(S_{\alpha}{ }^{\prime}\right)^{(i)}=(-1)^{|\alpha|} S_{\alpha}^{(i)} ; \quad i=1,3 \\
& \left(S_{\alpha}{ }^{\prime}\right)^{(2)}=S_{\alpha}^{(2)}
\end{aligned}
$$

There is a local unitary operator taking the $S_{\alpha}$ into the $S_{\alpha}{ }^{\prime}$ (rotations by $\pi$ about the $y$ axis for $|\boldsymbol{\alpha}|$ odd). Moreover, the Hamiltonian for the $S^{\prime}$ system is ferromagnetic in the 1 and 3 variables, which have real representatives, and antiferromagnetic in the 2 variable, which has an imaginary representative. Using Lemma 6.1 and the method of the proof of Theorems 4.1 and 4.2 , we see that

$$
\left(b_{\mathrm{p}}\right)^{(i)} \leqslant 1 / 2 E_{\mathbf{p}}
$$

for $i=1,3$. But $\left(b_{\mathbf{p}}\right)^{(i)}=b_{\mathbf{p}^{\prime}}^{(i)}$ for $i=1,3$, so we have that

$$
b_{\mathrm{p}}^{(3)} \leqslant 1 / 2 E_{\mathrm{p}}{ }^{\prime}
$$

By symmetry

$$
\sum_{i} b_{\mathbf{p}}^{(i)} \leqslant 3 / 2 E_{\mathbf{p}}{ }^{\prime}
$$

To estimate $C_{\mathbf{p}}$, we introduce a basic constant $\rho_{\nu}$ as follows. Let $H_{\Lambda}$ be the Hamiltonian of the antiferromagnet with periodic boundary conditions. We define

$$
\rho_{v}=-\lim _{\Lambda \rightarrow \infty}(\nu|\Lambda|)^{-1} \inf \operatorname{spec}\left(H_{\Lambda}\right)
$$

where one can show the limit exists as $\Lambda \rightarrow \infty$ in a suitable (e.g., van Hove) 
sense by mimicking the standard arguments on the existence of the thermodynamic limit for the free energy. ${ }^{(41)}$ By (55) we have that

$$
C_{\mathbf{p}} \leqslant 4 E_{\mathrm{p}} \rho_{\mathrm{v}}
$$

Thus (49) becomes

$$
S(S+1)>\sqrt{\frac{3}{2}}\left(\rho_{v}\right)^{1 / 2}(2 \pi)^{-v} \int_{\left|p_{\mid}\right| \leq \pi}\left(E_{\mathbf{p}} / E_{\mathbf{p}}\right)^{1 / 2} d^{v} p
$$

If $\nu \geqslant 3$ [so that (50) holds] and (62) holds, then a lower bound on the critical $\beta$ is given by the solution of

$$
S(S+1)=(2 \pi)^{-v} \int_{\left|p_{i}\right| \leq \pi}\left(E_{\mathbf{p}} / E_{\mathbf{p}}\right)^{1 / 2}\left(3 \rho_{v} / 2\right)^{1 / 2} \operatorname{coth}\left[\beta\left(2 \rho_{v} E_{\mathbf{p}} E_{\mathbf{p}}{ }^{\prime} / 3\right)^{1 / 2}\right] d \nu_{p}
$$

We conclude this section by demonstrating that (62) holds for $v \geqslant 3$, $S \geqslant 1$; and when $S=1 / 2$, for $\nu$ sufficiently large. To do this we use the bound of Anderson ${ }^{(1)}$ (reproduced in Appendix C):

$$
\rho_{\nu} \leqslant S\left[S+(2 \nu)^{-1}\right]
$$

Moreover, by the Schwarz inequality

$$
\nu K_{v}=(2 \pi)^{-v} \int_{\left|p_{j}\right| \leq \pi}\left(E_{\mathbf{p}} / E_{\mathbf{p}}\right)^{1 / 2} d^{v} p \leqslant\left[\nu G_{\nu}(0)\right]^{1 / 2}
$$

[where $G_{\nu}(0)$ is given by (11)], so that (62) certainly holds if

$$
S(S+1)^{2}>\frac{3}{2}\left[S+(2 \nu)^{-1}\right] \nu G_{\nu}(0)
$$

Using the fact that $\nu G_{v}(0)$ is monotone decreasing in $\nu$ (see the next section) and that $G_{3}(0)=0.505 . . .{ }^{(45)}$ we see that (66) certainly holds if $S \geqslant 1$ and $\nu \geqslant 3$. Moreover, since $\nu G_{v}(0) \rightarrow 1$ as $\nu \rightarrow \infty$, even for $S=1 / 2$, (66) holds for $\nu$ sufficiently large-in fact, (62) is so close to holding for $\nu=3, S=1 / 2$ when (64) is used that we expect that numerical analysis of $\nu K_{\nu}$ for $\nu \geqslant 4$ would show that, using (64) to bound $\rho_{y},(62)$ holds for $\nu \geqslant 4, S=1 / 2$. It is also true that $\nu K_{v}$ is monotone decreasing in $\nu$ by the method of Theorem 7.1, because $f(x)=[(1+x) /(1-x)]^{1 / 2}$ is convex in $[0,1)$. that

M. L. Glasser has kindly evaluated the integral in (62) for us and found

$$
3 K_{3}=1.157
$$

[to be compared with the bound 1.23 of (65)]. Thus for (62) to hold with $S=1 / 2, \nu=3$ one needs that $\rho_{3}<0.28$. The best rigorous bounds [from (64) and the trivial $\rho_{v} \geqslant S^{2}$ ] are $0.25<\rho_{3}<0.33$. The best numerical 
estimate on $\rho_{3}$ we can find is $\rho_{3} \cong 0.3{ }^{(22)}$ but there is a need for better rigorous bounds to be certain that (62) fails for $\nu=3, S=1 / 2$ and to check the $v$ for which (62) holds when $S=1 / 2$. We summarize with:

Theorem 6.2. The nearest neighbor, simple cubic antiferromagnet has a phase transition at sufficiently low temperature if $\nu \geqslant 3, S=1,3 / 2, \ldots$ or if $S=1 / 2$ and $\nu$ is sufficiently large.

\section{DIMENSIONAL DEPENDENCE OF THE CRITICAL TEMPERATURE}

It is a well-known element of folklore that as $\nu \rightarrow \infty$, transition temperatures approach those of the mean field approximation. Our goal in this final section is to note the extent to which this piece of folklore is proven by the upper bounds on the transition $\beta$ of FSS for the classical $N$-vector model and our putative bounds for the spin- $S$ quantum Heisenberg ferromagnet. For the classical case the results are summarized in Table III. In the third column we give Griffiths' ${ }^{(18)}$ rigorous lower bound for the Ising-model critical $\beta$ and in the fourth column we give the lower bound obtained by applying the method of Brascamp and Lieb ${ }^{(6)}$ to the case at hand (see Appendix D). The table should be supplemented with the following result:

Theorem 7.1. $\nu G_{v}(0)$ is monotone decreasing and approaches 1 as $\nu \rightarrow \infty$.

Proof. By the convexity of the function $g(x)=x^{-1}$ for $x \geqslant 0$, we have that for $f_{1}, \ldots, f_{n} \geqslant 0$ and $F=\sum_{j=1}^{n} f_{j}$

$$
F^{-1} \leqslant \sum_{j=1}^{n} n^{-2}(n-1)\left(F-f_{j}\right)^{-1}
$$

since

$$
F=\sum_{j=1}^{n} \frac{1}{n} \frac{n}{n-1}\left(F-f_{j}\right)
$$

Table III. Transition $\beta$ in $N$-Vector Model

\begin{tabular}{ccccc}
\hline$N$ & $\begin{array}{c}\text { FSS upper } \\
\text { bound }\end{array}$ & $\begin{array}{c}\text { Griffiths' lower } \\
\text { bound }\end{array}$ & BL lower bound & Mean field \\
\hline 1 & $\frac{1}{2} G_{v}(0)$ & $\tanh ^{-1}(2 v)^{-1}$ & $1 / 4 \nu$ & $1 / 2 \nu$ \\
$\geqslant 2$ & $\frac{1}{2} N G_{\nu}(0)$ & - & $N / 4 \nu$ & $N / 2 \nu$ \\
\hline
\end{tabular}


Taking $f_{j}=1-\cos p_{j}$ and integrating, we obtain

$$
G_{n}(0) \leqslant n^{-1}(n-1) G_{n-1}(0)
$$

proving the required monotonicity.

That the limit is 1 follows from the lemma below.

Lemma 7.1. Suppose that $f_{1}, \ldots, f_{n}, \ldots$ are identically distributed, independent, nonnegative random variables and that $F$ is a function on $(0, \infty)$ such that:

(i) $|F(x+\epsilon)-F(x)| \leqslant C \epsilon / x^{k}$, all $x>0$, and $0<\epsilon<1$, for some $C, k>0$.

(ii) $\operatorname{Exp}\left[\left(\sum_{i=1}^{m} f_{i}\right)^{-k}\right]<\infty$ for some $m$, where $\operatorname{Exp}=$ "expectation." Then

$$
\lim _{n \rightarrow \infty} \operatorname{Exp}\left[F\left(\frac{1}{n} \sum_{i=1}^{n} f_{i}\right)\right]=F(\operatorname{Exp}(f))
$$

Proof. By the convexity of $1 / x^{k}$ and the argument above, $\operatorname{Exp}\{[1 / n$ $\left.\left.\sum_{i=1}^{n} f_{i}\right]^{-k}\right\}$ is monotone decreasing in $n$. Thus, for $n \geqslant m$ and $0<\varepsilon<1$

$$
\begin{aligned}
& \left|\operatorname{Exp}\left[F\left(\frac{1}{n} \sum_{i=1}^{n}\left(f_{i}+\epsilon\right)\right)\right]-\operatorname{Exp}\left[F\left(\frac{1}{n} \sum_{i=1}^{n} f_{i}\right)\right]\right| \\
& \leqslant C \epsilon \operatorname{Exp}\left[\left(\sum_{i=1}^{m} f_{i}\right)^{-k} m^{k}\right]
\end{aligned}
$$

Moreover, by the usual strong law of large numbers ${ }^{(7)}$

$$
\operatorname{Exp}\left[F\left(\frac{1}{n} \sum_{i=1}^{n}\left(f_{i}+\epsilon\right)\right)\right] \rightarrow F(\operatorname{Exp}(f)+\epsilon)
$$

These two formulas and the continuity of $F$ imply the result.

Given this theorem, we see that the ratio of the FSS bound to the mean field theory bound approaches 1 . Since the Griffiths bound also approaches mean field theory, we have the following theorem:

Theorem 7.2. Let $T_{c}{ }^{v}$ be the true transition temperature of the simple cubic, $\nu$-dimensional Ising model. Then

$$
T_{c}^{y} / 2 \nu \rightarrow 1
$$

as $\nu \rightarrow \infty$.

Unfortunately (for reasons we explain in Appendix D), the BrascampLieb bound appears to be off by a factor of two in the nearest neighbor case, so for $N \geqslant 2$, where the only upper bound we have on $T_{c}$ is theirs, we cannot 
prove that $N T_{c}{ }^{v} / 2 v$ converges to 1 . We do know, however, that its $\lim$ is $\geqslant 1$ and its $\overline{\lim }$ is $\leqslant 2$, so that the FSS bound certainly has the right $\nu$ dependence and if mean field theory is asymptotically correct, then FSS is asymptotically correct.

To discuss the $\nu$ dependence of our presumed upper bound on the critical $\beta$ in the spin- $S$ quantum Heisenberg model, we abstract the argument of Theorem 7.1:

Theorem 7.3. Let $f_{1}, \ldots, f_{n}, \ldots$ be identically distributed, independent random variables. Let $F$ be a nonnegative convex (respectively concave) function on the convex hull of $\operatorname{Ran} f_{1}$. Then $\operatorname{Exp}\left[F\left((1 / n) \sum_{i=1}^{n} f_{i}\right)\right]$ is monotone decreasing (respectively increasing) in $n$.

Proof. Use the convexity and

$$
\frac{1}{n} \sum_{i=1}^{n} f_{i}=\frac{1}{n} \sum_{j=1}^{n}\left(\frac{1}{n-1} \sum_{\substack{i \neq j \\ 1 \leqslant i \leqslant n}} f_{i}\right)
$$

Theorem 7.4. Let $\widetilde{\beta}_{c}(S, \nu)$ be the solution of $(52 H)$ and $\zeta(S, \nu)=$ $\nu \widetilde{\beta}_{c}(S, \nu)$. Let $\zeta(S, \infty)$ be the solution of the equation

$$
S+1=\sqrt{\frac{3}{2}} \operatorname{coth}\left[\sqrt{\frac{2}{3}} S \zeta(S, \infty)\right]
$$

(tabulated in Table IV). Then $\zeta(S, \nu)$ is monotone decreasing to $\zeta(S, \infty)$ as $\nu \rightarrow \infty$.

Proof. Let $f_{i}(i=1,2, \ldots)$ be the function $1-\cos p_{i}$ on $[-\pi, \pi]^{\infty}$ with the natural product probability measure $X d p_{i} / 2 \pi$. Let $F$ be the function

$$
F(x)=\sqrt{\frac{3}{2}} \operatorname{coth}\left(\sqrt{\frac{2}{3}} x\right)
$$

Table IV. Values of ${ }^{a} \zeta(S, \infty)=$

$$
\lim _{v \rightarrow \infty} \nu \tilde{\beta}_{c}(S, \nu)
$$

\begin{tabular}{rlc}
\hline$S$ & $S^{2} \zeta(S, \infty)$ & $S(S+1) \zeta(S, \infty)$ \\
\hline$\frac{1}{2}$ & 0.702 & 2.11 \\
1 & 0.873 & 1.75 \\
$\frac{3}{2}$ & 0.985 & 1.64 \\
2 & 1.06 & 1.59 \\
$\frac{5}{2}$ & 1.12 & 1.57 \\
3 & 1.16 & 1.55 \\
$\frac{7}{2}$ & 1.20 & 1.54 \\
4 & 1.22 & 1.53 \\
15 & 1.41 & 1.50 \\
50 & 1.47 & 1.50 \\
$\infty$ & 1.50 & 1.50 \\
\hline
\end{tabular}

${ }^{a} \nu$ is the lattice dimension; $S$ is the spin. 
Then $\zeta(S, \nu)$ solves

$$
S+1=\operatorname{Exp}\left[F\left(S \zeta(S, \nu) \nu^{-1} \sum_{i=1}^{v} f_{i}\right)\right]
$$

Now $F$ is convex and strictly monotone decreasing (see Appendix A). So, using Theorem 7.3,

$$
\begin{aligned}
\operatorname{Exp}[ & \left.F\left(S \zeta(S, \nu-1) \nu^{-1} \sum_{i=1}^{v} f_{i}\right)\right] \\
& \leqslant \operatorname{Exp}\left[F\left(S \zeta(S, \nu-1)(\nu-1)^{-1} \sum_{i=1}^{\nu-1} f_{i}\right)\right] \\
& =S+1=\operatorname{Exp}\left[F\left(S \zeta(S, \nu) \nu^{-1} \sum_{i=1}^{\nu} f_{i}\right)\right]
\end{aligned}
$$

By the monotonicity of $F, \zeta(S, v-1) \geqslant \zeta(S, v)$. Thus $\zeta$ approaches a limit $\zeta_{\infty}$. By a simple extension of Lemma $7.1, \zeta_{\infty}$ solves (67) and so equals $\zeta(S, \infty)$.

Mean field theory gives the same transition temperature for the spin- $S$ Heisenberg model and the spin- $S$ Ising model. Thus the $\zeta(S, \infty)$ result does not agree with mean field theory, but at least the gross (i.e., $\nu^{-1}$ ) structure of mean field theory and our bound agree.

\section{APPENDIX A. A GARDEN OF COTH AND TANH}

In this appendix, we collect some basic properties of the functions coth $x$ and $f$ given by $f(x \tanh x)=x^{-1} \tanh x$.

Theorem A.1. The function

$$
g(x)=\operatorname{coth} x
$$

is strictly monotone decreasing and convex on $[0, \infty)$. The function

$$
h(x)=g(x)-x^{-1}
$$

is strictly monotone increasing and concave on $[0, \infty)$ with $h(0)=0$. The function $g(x)$ obeys

$$
\begin{gathered}
x^{-1}<g(x)<1+x^{-1}, \quad x>0 \\
|g(x+\epsilon)-g(x)| \leqslant \epsilon / x^{2}, \quad 0<\epsilon, \quad 0<x
\end{gathered}
$$

Proof. By direct calculation, $g^{\prime}(x)=-(\sinh x)^{-2}$ and $g^{\prime \prime}(x)=2(\cosh x)$ $(\sinh x)^{-3}$. The first sentence is then obvious. The bound $\sinh x>x$ (which comes from the power series for $\sinh$ ) then yields $0 \geqslant g^{\prime}(x)>-x^{-2}$, from 
which (A2) follows, as does $h^{\prime}>0$. Now $\lim _{x \rightarrow 0} h(x)=0$ is obvious from the power series expansion of coth and sinh. Condition (A1) just says $h(0)<h(x)<h(\infty)$. This leaves the concavity of $h$. Given $g^{\prime \prime}$ above, we need that

$$
x^{3} \cosh x \leqslant \sinh ^{3} x
$$

or equivalently that

$$
\frac{\sinh 2 x}{2 x} \leqslant\left(\frac{\sinh x}{x}\right)^{4}
$$

or

$$
\prod_{n=1}^{\infty}\left[1+(2 x / n \pi)^{2}\right] \leqslant \prod_{n=1}^{\infty}\left[1+(x / n \pi)^{2}\right]^{4}
$$

which is obvious.

Remark. The convexity of $g$ and concavity of $h$ are useful in obtaining quick (i.e., hand calculator!) estimates on the function

$$
H_{\nu}(x)=(2 \pi)^{-v} \int_{\left|p_{i}\right| \leqslant \pi} \operatorname{coth}\left(x E_{\mathrm{p}}\right) d^{v} p
$$

For example, they lead to the bounds

$$
\operatorname{coth}(\nu x) \leqslant H_{\nu}(x) \leqslant \operatorname{coth}(\nu x)+(\nu x)^{-1}\left[\nu G_{\nu}(0)-1\right]
$$

via Jensen's inequality and the fact that $(2 \pi)^{-v} \int_{\left|p_{i}\right| \leqslant n} E_{\mathbf{p}} d^{v} p=\nu$.

Now we turn to the study of the function given by

$$
f(x \tanh x)=x^{-1} \tanh x
$$

Theorem A.2. $f$ is a well-defined, strictly monotone-decreasing, convex function of $(0, \infty)$ to $(0,1)$ with $\lim _{x \rightarrow 0} f(x)=1$ and $\lim _{x \rightarrow \infty} f(x)=0$.

Proof. Let $\alpha(x)=x \tanh x$. Since $\alpha$ is strictly monotone increasing from $(0, \infty)$ to $(0, \infty)$, it has an inverse function $\beta(x)$ which is also strictly monotone increasing. Let $\gamma(x)=x^{-1} \tanh x$. Then $\gamma^{\prime}(x)=x^{-1}(\cosh x)^{-2}-$ $x^{-2} \tanh x=(x \cosh x)^{-1}\left[(\cosh x)^{-1}-x^{-1} \sinh x\right]<0$ since $(\cosh x)^{-1}$ $<1$ and $x^{-1} \sinh x>1$. Thus $f=\gamma \circ \beta$ is strictly monotone decreasing. Since $\beta(0)=0, \beta(\infty)=\infty$ and $\gamma(0)=1, \gamma(\infty)=0$, we have the limiting statements.

This leaves the proof of the convexity of $f$. Our original proof involved a straightforward but rather brutal computation. V. Bargmann provided us with the following proof, which, while not free of complexity, is a con- 
siderable improvement. We first note that for a function $G$ on $(0, \infty)$ to be convex, it is necessary and sufficient that for any $0<x<y<z$

$$
\operatorname{det}\left|\begin{array}{lll}
1 & x & G(x) \\
1 & y & G(y) \\
1 & z & G(z)
\end{array}\right| \geqslant 0
$$

for, if $H$ is the linear function with $H(x)=G(x), H(z)=G(z)$, the determinant in (A7) is $[H(y)-G(y)](z-x)$ and convexity is that $G(y)<H(y)$. Thus convexity of $f$ is equivalent to

$$
\operatorname{det}\left|\begin{array}{lll}
1 & x \tanh x & x^{-1} \tanh x \\
1 & y \tanh y & y^{-1} \tanh y \\
1 & z \tanh z & z^{-1} \tanh z
\end{array}\right| \geqslant 0
$$

for $x \leqslant y \leqslant z$, where we have used the monotonicity of $\alpha$. Multiplying by $x y z(\operatorname{coth} x)(\operatorname{coth} y)(\operatorname{coth} z)$ and interchanging columns, we find that (A8) is equivalent to

$$
\operatorname{det}\left|\begin{array}{ccc}
1 & x^{2} & x \operatorname{coth} x \\
1 & y^{2} & y \operatorname{coth} y \\
1 & z^{2} & z \operatorname{coth} z
\end{array}\right| \leqslant 0
$$

Again using the criterion (A7), we see that (A9) is equivalent to the concavity of the function $P(x)=x^{1 / 2} \operatorname{coth}\left(x^{1 / 2}\right)$.

We have therefore shown the convexity of $f$ is equivalent to the concavity of $P$. By a straightforward calculation,

$$
\begin{aligned}
4 y^{3}\left(\sinh ^{3} y\right) P^{\prime \prime}\left(y^{2}\right) & =2 y^{2} \cosh y-\cosh y \sinh ^{2} y-y \sinh y \\
& =y(y \cosh y-\sinh y)+(\cosh y)\left(y^{2}-\sinh ^{2} y\right) \\
& \leqslant y(y \cosh y-\sinh y)+(\cosh y)\left(-\frac{y^{4}}{3}\right) \\
& =\sum_{n=0}^{\infty} y^{2 n+4}\left[\frac{1}{(2 n+1)(2 n+3)}-\frac{1}{3}\right] \frac{1}{(2 n) !} \leqslant 0
\end{aligned}
$$

In the third step, we used

$$
-\left(y^{2}-\sinh ^{2} y\right)=(y+\sinh y)(\sinh y-y) \geqslant(2 y)\left(y^{3} / 6\right) .
$$

Theorem A.3. Let $F$ be any convex function on $[0, \infty)$. The function $H(y, c)=y\left[F\left(c y^{-1}\right)-1\right]$ for $y, c>0$ is jointly convex in $(y, c)$. If $F$ is monotone decreasing, then $H$ is monotone decreasing in both $y$ and $c$. 
Proof. The second derivative matrix

$$
F^{\prime \prime}\left(\frac{c}{y}\right)\left(\begin{array}{cc}
c^{2} y^{-3} & -c y^{-2} \\
-c y^{-2} & y^{-1}
\end{array}\right)
$$

is positive semidefinite. For $y$ fixed, $H$ is monotone decreasing as $c$ increases since $F$ is monotone decreasing. For $c$ fixed, $\partial H / \partial y=F\left(c y^{-1}\right)-1-$ $c y^{-1} F^{\prime}\left(c y^{-1}\right)$ is negative by the convexity of $F$ [which implies that $(0, F(0))$ must lie above the tangent to $F$ at the point $\left.\left(c y^{-1}, F\left(c y^{-1}\right)\right)\right]$.

Theorem A.4. ${ }^{6}$

$$
f(x) \geqslant x^{-1}\left(1-e^{-x}\right)
$$

Remark. As we explained in Section 3, this can also be proved by appealing to the best possible nature of our bound there and to the bound of Roepstorff.

Proof. In (A10) replace $x$ by $(x / 2) \tanh (x / 2)=(x / 2)\left(e^{x}-1\right) /\left(e^{x}+1\right)$. Then (A10) is equivalent to

$$
\frac{2}{x} \frac{e^{x}-1}{e^{x}+1} \geqslant \frac{2}{x} \frac{e^{x}+1}{e^{x}-1}\left[1-\exp \left(-\frac{x}{2} \frac{e^{x}-1}{e^{x}+1}\right)\right]
$$

or

$$
4 e^{x}\left(e^{x}+1\right)^{-2} \leqslant \exp \left(-\frac{x}{2} \frac{e^{x}-1}{e^{x}+1}\right)
$$

or

$$
x-2 \ln \frac{e^{x}+1}{2} \leqslant-\frac{x}{2} \frac{e^{x}-1}{e^{x}+1}
$$

or

$$
Q(x) \equiv x \frac{3 e^{x}+1}{e^{x}+1}-4 \ln \frac{e^{x}+1}{2} \leqslant 0
$$

Now, by a simple computation, $Q(0)=0$ and

$$
Q^{\prime}(x)=2 e^{x}\left(e^{x}+1\right)^{-2}(x-\sinh x)<0
$$

so (A11) holds.

\section{APPENDIX B. COMPUTATIONS WITH HARMONIC OSCILLATORS}

Let $H=\omega A^{*} A$ with $\left[A, A^{*}\right]=s$, where $s>0$ is the $c$-number commutator, in a space where there is a vector $\psi_{0}$ with $A \psi_{0}=0$ and $\left\{\left(A^{*}\right)^{n} \psi_{0}\right\}$ a spanning set. Then there is an orthonormal basis $\psi_{n}$ with $H \psi_{n}=n \omega s \psi_{n}$ ${ }^{6}$ See footnote 5, p. 349. 
and $\left(\psi_{m}, A \psi_{n}\right)=\delta_{m, n-1}(n s)^{1 / 2}$. Thus, using (21) when $\beta=1$, it is easy to verify that

$$
\left(A^{*}, A\right)=\omega^{-1}
$$

Moreover,

$$
\frac{1}{2}\left\langle\left(A^{*} A+A A^{*}\right)\right\rangle=\left\langle A^{*} A+\frac{1}{2} s\right\rangle=\frac{1}{2} s+\omega^{-1}\langle H\rangle=\frac{1}{2} s \operatorname{coth}\left(\frac{1}{2} \omega s\right)
$$

Finally,

$$
\left[\left[A^{*}, H\right], A\right]=\omega s^{2} \quad(c \text {-number })
$$

so, in particular,

$$
\frac{\left\langle\left[\left[A^{*}, H\right], A\right]\right\rangle}{4\left(\frac{1}{2}\left\langle A^{*} A+A A^{*}\right\rangle\right)}=\frac{\omega S}{2} \tanh \left(\frac{\omega S}{2}\right)
$$

There are three important things to notice about the formulas $(B 1)-\left(\mathbf{B}^{\prime}\right)$ :

(i) Of the various quantities, only the Duhamel two-point function is independent of the commutator parameter $s$. This adds to the evidence elsewhere in this paper that the DTF is "closer" to the classical two-point function than the usual thermal two-point function.

(ii) The inequality of Section 3

$$
\left(A^{*}, A\right) \geqslant \frac{1}{2}\left\langle A^{*} A+A A^{*}\right\rangle f\left(\left\langle\left[\left[A^{*}, H\right], A\right]\right\rangle /\left(4 \cdot \frac{1}{2}\left\langle A^{*} A+A A^{*}\right\rangle\right)\right)
$$

is saturated in the case at hand, and, as $\omega$ and/or $s$ vary, the argument of $f$ runs through all values from 0 to $\infty$. This implies that $f$ is the best possible function.

(iii) The Bogoliubov inequality in its strong form $(\beta=1)$

$$
|\langle[A, C]\rangle|^{2} \leqslant\left(A^{*}, A\right)\left\langle\left[C^{*},[H, C]\right]\right\rangle
$$

is saturated in the example at hand if $C$ is chosen to be $A^{*}$.

Not only are the inequalities of Section 3 saturated by a single harmonic oscillator, but those of Section 4 are saturated by a system of coupled harmonic oscillators. This is not surprising since the classical analogs of these inequalities $^{(12)}$ are saturated by classical harmonic oscillators. For, in a $\nu$-dimensional box $\Lambda$, let

$$
H=\frac{1}{2} \sum_{\alpha \in \Lambda}\left(p_{\alpha}^{2}+x_{\alpha}^{2}\right)-J \sum_{|\alpha-\beta|=1} x_{\alpha} x_{\beta}
$$

on $L^{2}\left(\mathbb{R}^{|\Lambda|}\right)$ with $p_{\alpha}=(1 / i) \partial / \partial x_{\alpha}$. By using normal modes (which are just Fourier series), it is easy to see that for $H$ to be positive one needs

$$
2 J_{\nu}<1
$$

[for the frequencies obey $\mu(\mathbf{k})=1-2 J \sum_{i=1}^{v} \cos k_{i}$ ] and

$$
\left(\hat{x}(\mathbf{k})^{*}, \hat{x}(\mathbf{k})\right)=[\beta \mu(\mathbf{k})]^{-1}
$$


As $J$ approaches $(2 v)^{-1}$ [the largest value allowed by (B4)], the inequality of Section 4 [in which the right side of (B5) is replaced by $\left(2 J E_{\mathbf{k}}\right)^{-1}$ ] is saturated.

The saturation of the inequalities from Sections 3 and 4 means the presumed lower bound on the transition temperature given by $(52 H)$ will agree with the transition temperature in a spin wave theory (which is essentially a harmonic approximation) with three degrees of freedom at each site instead of the usual two.

\section{APPENDIX C. LOWER BOUNDS ON THE GROUND-STATE ENERGIES OF SOME QUANTUM SPIN HAMILTONIANS}

In this appendix, we wish to derive lower bounds on the ground-state energy of the Hamiltonian of the $x-y$ model and of the isotropic Heisenberg antiferromagnet. These bounds are needed, respectively, in Sections 5 and 6 to obtain upper bounds on $|\Lambda|^{-1}\left\langle-H_{\Lambda}\right\rangle$. At first sight one might expect the $x-y$ model to look more like the Heisenberg ferromagnet, which has an exactly calculable ground state, rather than the antiferromagnet. This expectation is wrong, as can be understood by making a $180^{\circ}$ rotation about the $z$ axis at each site in one of the natural sublattices of the $x-y$ ferromagnet.

We shall prove the following result:

Theorem C.1. For $n+1$ independent spin- $1 / 2$ spins $\mathbf{S}_{0}, \ldots, \mathbf{S}_{n}$ the maximum eigenvalue of

$$
A_{n+1}=S_{0}^{(x)} \sum_{i=1}^{n} S_{i}^{(x)}+S_{0}^{(y)} \sum_{i=1}^{n} S_{i}^{(y)}
$$

is $\frac{1}{2} l$ if $n=2 l-1$ and $\frac{1}{2}[l(l+1)]^{1 / 2}$ if $n=2 l$.

Theorem C.2 (Anderson ${ }^{(1)}$ ). For $n+1$ independent spin-S spins $\mathbf{S}_{0}, \ldots, \mathbf{S}_{n}$, the maximum eigenvalue of

$$
B_{n+1}=-\mathbf{S}_{0} \cdot \sum_{i=1}^{n} \mathbf{S}_{i}
$$

is $S(n S+1)$.

Theorem C.2 and the proof we give here for the reader's convenience are due to Anderson. ${ }^{(1)}$ Both lead directly to upper bounds on $|\Lambda|^{-1}\left\langle-H_{\Lambda}\right\rangle$ since $-H_{\Lambda}$ can be written as a sum of $|\Lambda|$ operators unitarily equivalent to $\frac{1}{2} A_{n+1}\left(\right.$ resp. $\left.\frac{1}{2} B_{n+1}\right)$ where $n=2 \nu$.

Proof of Theorem C.1. We first note that for two spins, with the usual up-down notation,

$$
\begin{aligned}
& A_{2}|++\rangle=0, \quad \quad A_{2} \mid-->=0 \\
& \left.A_{2}\left|+->=\frac{1}{2}\right|-+\right\rangle, \quad A_{2}|-+\rangle=\frac{1}{2} \mid+->
\end{aligned}
$$


Since $A_{n}$ is a sum of pair operators equivalent to $A_{2}, A_{n}$ is also a matrix with nonnegative elements. By the Perron-Frobenius theorem, the largest eigenvalue has an associated eigenvector with nonnegative coefficients in the up-down basis (see Lieb and Mattis ${ }^{(25,26)}$ ). Moreover, if we decompose the Hilbert space into $n+2$ pieces $\mathscr{H}_{j}$ corresponding to $j$ up spins, $A_{n}$ leaves each piece invariant and the matrix is ergodic on each $\mathscr{H}_{j}$. Thus, on $\mathscr{H}_{j}$ the largest eigenvalue is simple and has strictly positive coefficients. In $\mathscr{H}_{j}$, there are $\left({ }_{j}^{n} \begin{array}{l}n \\ 1\end{array}\right)$ basis vectors with $S_{0}^{(3)}=+\frac{1}{2}$ (call their sum $\phi$ ) and $\left(\begin{array}{l}n \\ j\end{array}\right)$ with $S_{0}^{(3)}=-\frac{1}{2}$ (call their sum $\psi$ ). By the above remarks, the eigenvector corresponding to the maximum eigenvalue $\lambda_{j}$ is of the form $\eta=a \phi+b \psi$.

Now, given the action of $A_{2}, A_{n}$ applied to a basis vector summand in $\phi$ yields $\frac{1}{2}$ times the sum of $n-j+1$ vectors, which are summands in $\psi$. By symmetry it follows that

$$
A_{n} \phi=\frac{1}{2}(n-j+1)\left[\left(\begin{array}{c}
n \\
j-1
\end{array}\right) /\left(\begin{array}{l}
n \\
j
\end{array}\right)\right] \psi=\frac{1}{2} j \psi
$$

and similarly

$$
A_{n} \psi=\frac{1}{2} j\left[\left(\begin{array}{l}
n \\
j
\end{array}\right) /\left(\begin{array}{c}
n \\
j-1
\end{array}\right)\right]=\frac{1}{2}(n+1-j) \phi
$$

The equation $A_{n} \eta=\lambda_{j} \eta$ thus becomes $\frac{1}{2} j a=\lambda_{j} b$ and $\frac{1}{2}(n+1-j) b=\lambda_{j} a$, so that $\lambda_{j}{ }^{2}=\frac{1}{4}(n+1-j) j$. The maximum value of $\lambda_{j}$ clearly occurs for $j=l$ when $n=2 l$ or $2 l-1$.

Proof of Theorem C.2 (following Anderson ${ }^{(1)}$ ). This is an exercise in addition of angular momentum since $B_{n+1}=-S_{0} \cdot J$ with $J=\sum_{i=1}^{n} \mathbf{S}_{i}$. If $j$ is the eigenvalue of $J$ and $\alpha=\min (S, j)$, then the maximum value of $B_{n+1}$ is

$$
-\frac{1}{2}[|S-j|(|S-j|+1)-S(S+1)-j(j+1)]=j S+\alpha
$$

Among the values $j=0, \ldots, n S$, the maximum value of this occurs when $j=n S$.

\section{APPENDIX D. ON THE BRASCAMP-LIEB UPPER BOUND}

In Ref. 6, Brascamp and Lieb applied a basic theorem on log concave function to give upper bounds on transition temperatures in pair interacting Ising models (with no restriction that the interaction be either ferromagnetic or nearest neighbor). In this appendix, we extend this result to the classical $N$-vector model. We begin with an abstraction of the Brascamp-Lieb argument: 
Theorem D.1. Let $d \mu$ be a measure on $\mathbb{R}^{N}$ with support on $\{\mathbf{s}|| \mathbf{s} \mid=1\}$. Define the convex function $f$ on $\mathbb{R}^{N}$ by

$$
f(\mathbf{x})=\log \int \exp (\mathbf{x} \cdot \mathbf{s}) d \mu(\mathbf{s})
$$

Let $a=\max _{x}\left(\partial^{2} f / \partial x_{i} \partial x_{j}\right)_{\infty}$, where $\left(A_{i j}\right)_{\infty}$ for a matrix $A_{i j}$ indicates its largest eigenvalue. Hence $-\frac{1}{2} a \mathbf{x}^{2}+f(\mathbf{x})$ is concave in $\mathbb{R}^{N}$. Let $\Gamma$ be a lattice in $\mathbb{R}^{v}$ (i.e., discrete subgroup of $\mathbb{R}^{v}$ ) and let $J$ be a function on $\Gamma$ with $\sum_{\alpha \in \Gamma}|J(\alpha)|<\infty$ and $J(-\alpha)=J(\alpha)$. Let $J_{\max }\left(\right.$ resp. $\left.J_{\min }\right)$ denote the largest (resp. smallest) point of the spectrum of the operator

$$
(\mathscr{J} x)_{\alpha}=\sum_{\beta} J_{\alpha-\beta} x_{\beta}
$$

on $l^{2}(\Gamma)$. Then, if

$$
\beta\left(J_{\max }-J_{\min }\right)<a^{-1}
$$

there is no long-range order in the model with finite-volume partition function:

$$
Z_{\Lambda}=\int \prod_{\alpha \in \Lambda} d \mu\left(\mathbf{s}_{\alpha}\right) \exp \left(\frac{1}{2} \beta \sum_{\alpha, \beta \in \Lambda} J_{\alpha \beta} \mathbf{s}_{\alpha} \cdot \mathbf{s}_{\beta}\right)
$$

Proof. Without loss, we can take $\beta=1$ by absorbing it into $J$. Let $\mathscr{J}^{\Lambda}$ denote the operator on $l^{2}(\Lambda)$ obtained from (D1) by restricting the sum to $\Lambda$. Choose $\epsilon$ small and $\Lambda$ large so that

$$
\epsilon+J_{\max }^{\Lambda}-J_{\min }^{\Lambda}<a^{-1}
$$

Let

$$
A_{\alpha \beta}^{\Lambda}=\left(\epsilon+J_{\max }^{\Lambda}\right) \delta_{\alpha \beta}-J_{\alpha-\beta}
$$

which is positive definite on $l^{2}(\Lambda)$. Moreover, $\exp \left[-\frac{1}{2} a \mathbf{x}^{2}+f(\mathbf{x})\right]$ is a $\log$ concave function, so, by the argument in Ref. 6,

$$
\sum_{\alpha, \beta \in \Lambda} z_{\alpha} z_{\beta}\left\langle s_{\alpha} s_{\beta}\right\rangle_{\Lambda} \leqslant \sum_{\alpha, \beta} z_{\alpha} z_{\beta} Q_{\alpha \beta}, \quad Q=a\left(1-a A^{\Lambda}\right)^{-1}
$$

for any $\mathrm{z}$ [by (D3), $1-a A^{\Lambda}$ is strictly positive definite]. Taking $\Lambda \rightarrow \infty$, we conclude the absence of long-range order.

Remark 1. By taking an Ising model with $J_{\alpha-\beta}=1$ if $|\alpha-\beta| \leqslant n$ and taking $n \rightarrow \infty$ ("mean field model"), one sees that (D2) is best possible in that $\beta\left(J_{\max }-J_{\min }\right)=(1+\epsilon) a^{-1}$ occurs with long-range order. However, for ferromagnetic Ising systems where $a=1, \beta \sum_{\alpha \neq 0} J_{\alpha}<1$ has no longrange order by a result of Griffiths. ${ }^{18}$ If $J$ is normalized so that $J(0)=0$, then $\sum_{\alpha \neq 0}\left|J_{\alpha}\right|=J_{\max }$ in the ferromagnetic case. For the nearest neighbor, simple cubic case, $J_{\max }-J_{\min }=4 v$ while $\sum_{\alpha \neq 0}\left|J_{\alpha}\right|=2 v$. As a result, (D2) is off by a factor of 2 at least in the Ising model, and we presume in general models, in this nearest neighbor case. 
Remark 2. The restriction that $d \mu$ have support on the sphere is easily removed by replacing $f$ by $\log \int \exp \left(\mathbf{x} \cdot \mathbf{s}+\beta J_{\max } \mathbf{s}^{2}\right) d \mu(\mathbf{s})$.

Theorem D.2. Let

$$
f(\mathbf{h})=\log \int_{\substack{|\mathbf{s}|=1 \\ \mathbf{s} \in \mathbb{R}^{N}}} \exp (\mathbf{h} \cdot \mathbf{s}) d \Omega_{\mathbf{s}}
$$

Then $\max _{\mathbf{h}}\left(\partial^{2} f / \partial h_{i} \partial h_{j}\right)_{\infty}=1 / N$.

Proof. When $\mathbf{h}=0,\left(\partial^{2} f / \partial h_{i} \partial h_{j}\right)_{\infty}=1 / N$ by inspection. By symmetry, $f(\mathbf{h})=F(h)$ is spherically symmetric. For $\mathbf{h} \neq \mathbf{0}$, it is easily seen that $\partial^{2} f / \partial h_{i} \partial h_{j}$ has eigenvalue $F^{\prime \prime}(h)$ once and $F^{\prime}(h) / h, N-1$ times. Clearly $F^{\prime}(0)=0$ by symmetry, so $F^{\prime}(h) / h \leqslant \max _{0 \leqslant x \leqslant h} F^{\prime \prime}(x)$ and thus

$$
\max _{\mathbf{h}}\left(\partial^{2} f / \partial h_{i} \partial h_{j}\right)_{\infty}=\max _{h} F^{\prime \prime}(h)
$$

We will prove that $F^{\prime \prime \prime}(h) \leqslant 0$ for $h \geqslant 0$ so that $\max F^{\prime \prime}(h)=F^{\prime \prime}(0)=1 / N$ [see, e.g., (D5) below]. We remark that the concavity of $F^{\prime}$ for $N \geqslant 3$ follows ${ }^{(32)}$ from general criteria of Ellis et al. ${ }^{(11)}$ for GHS inequalities [the distribution for $s_{1}$ is a limit of distributions of the form $\exp (-V)$ with $V=a_{2} x^{2}+a_{4} x^{4}+\cdots$, with $\left.a_{4}, a_{6}, \ldots \geqslant 0\right]$.

In terms of the obvious probability measure,

$$
\partial^{2} f / \partial h_{i} \partial h_{j}=\left\langle s_{i} s_{j}\right\rangle-\left\langle s_{i}\right\rangle\left\langle s_{j}\right\rangle, \quad \partial f / \partial h_{i}=\left\langle s_{i}\right\rangle
$$

Picking $\mathbf{h}=(h, 0, \ldots)$,

$$
\left\langle s_{1}{ }^{2}\right\rangle=d^{2} F / d h^{2}+(d F / d h)^{2}, \quad\left\langle s_{i}^{2}\right\rangle=h^{-1} d F / d h, \quad i \geqslant 2
$$

We conclude from $\left\langle\sum s_{i}{ }^{2}\right\rangle=1$ that $g(h) \equiv d F / d h$ obeys the differential equation

$$
g^{\prime}(h)+g(h)^{2}+(N-1) h^{-1} g(h)=1
$$

From (D4) and the fact that $g$ is odd we see that

$$
g(h)=N^{-1} h-N^{-2}(N+2)^{-1} h^{3}+O\left(h^{5}\right)
$$

so that $g^{\prime \prime}(h)<0$ for $h$ small. If $g^{\prime \prime}(h)<0$ is not true for all $h>0$, let $h_{0}$ be the first positive zero of $g^{\prime \prime}$. Now $g^{\prime}$ is positive ( $f$ is convex by Jensen's inequality) and $g$ is thus positive for all $h>0$. Multiplying (D4) by $h$ and taking two derivatives and using the fact that $g, g^{\prime} \geqslant 0$, we get

$$
h g^{\prime \prime \prime}+[(N+1)+2 h g] g^{\prime \prime}<0
$$

Since $h g$ is monotone and $g^{\prime \prime}<0$ on $\left(0, h_{0}\right)$, we have

$$
h g^{\prime \prime}+\alpha g^{\prime \prime} \leqslant 0, \quad 0<h<h_{0} ; \quad \alpha=N+1+2 h_{0} g\left(h_{0}\right)
$$

or

$$
\left(h^{\alpha} g^{\prime \prime}\right)^{\prime} \leqslant 0, \quad 0<h<h_{0}
$$


It follows that

$$
g^{\prime \prime}\left(h_{0}\right) \leqslant h_{0}^{-\alpha} h_{1}^{\alpha} g^{\prime \prime}\left(h_{1}\right)<0
$$

where $h_{1}$ is chosen so small that $g^{\prime \prime}\left(h_{1}\right)<0, h_{1}<h_{0}$. This contradiction proves that $F^{\prime \prime \prime}(h)<0$, so that $\max F^{\prime \prime}(h)=F^{\prime \prime}(0)=1 / N$.

Corollary D.1. In the nearest-neighbor, $N$-vector, $\nu$-dimensional ferromagnet, there is no long-range order in the two-point function if $\beta \leqslant N / 4 \nu$.

\section{APPENDIX E. TRANSFER MATRICES IN QUANTUM SPIN SYSTEMS}

The transfer matrix is a useful technique in classical spin systems, both as a calculational tool in one-dimensional systems and in the Onsager solution, ${ }^{(33,43)}$ and as a general theoretical tool (e.g., the appendix of Ref. 12). It is a well-known folk theorem that quantum spin systems do not possess transfer matrices. In this appendix, we want to show that this folk theorem is wrong, although the definition of our transfer matrix is sufficiently abstract that it is unlikely to be a useful calculational tool; it may turn out to be a useful theoretical tool. Our construction is borrowed from axiomatic and constructive quantum field theory, where a particular positivity condition has been emphasized by Osterwalder and Schrader ${ }^{(34)}$ in recovering the Hamiltonian semigroup from the Euclidean Green's functions. Klein ${ }^{(23)}$ has emphasized the idea of exploiting the notion of Osterwalder-Schrader positivity in more general contexts.

It should be emphasized that our transfer matrix differs from the usual one in an important way: Our basic inner product will have a lattice spacing built into it; that is, if $A, B$ are operators at a single site and $T$ is the transfer matrix, then the matrix element $\left(A, T^{n}(B)\right)$ will involve a thermal expectation of operators at sites a distance $n+1$ from each other.

We shall consider general Hamiltonians of the form (41) with real matrices. Consider a volume $\Lambda$ with $L_{1}=2 m_{1}, \alpha_{1}=-m_{1}+1,-m_{1}+2, \ldots, m_{1}$. Define an automorphism $R$ from operators on the sites with $\alpha_{1} \geqslant 1$ to the operators on the sites with $\alpha_{1} \leqslant 0$ by reflecting about the plane $\alpha_{1}=1 / 2$, e.g.,

$$
R\left(\mathbf{S}_{\alpha}\right)=\mathbf{S}_{\alpha^{\prime}}
$$

with $\alpha_{1}{ }^{\prime}=1-\alpha_{1}, \alpha_{2}{ }^{\prime}=\alpha_{2}, \ldots, \alpha_{v}{ }^{\prime}=\alpha_{v}$. Thus $H_{\Lambda}$ has the form

$$
H_{\Lambda}=B+R(B)-\sum_{i=1}^{k} C_{i} R\left(C_{i}\right)
$$

where $B, C_{1}, \ldots, C_{k}$ are operators on the sites with $\alpha_{1} \geqslant 1$. The first (resp. second) term represents the interactions to the right (resp. left) of $\alpha_{1}=1 / 2$, 
while the third term is the interaction across the plane $\alpha_{1}=1 / 2$. The minus sign in (E1), which restricts us to ferromagnets, will be crucial.

Theorem E.1. Suppose all matrices are real. For any operator $D$ on the sites with $\alpha_{1} \geq 1$,

$$
\langle R(D) D\rangle \geqslant 0
$$

Proof

$$
\begin{aligned}
\langle R(D) D\rangle= & {\left[\operatorname{Tr}\left(e^{-H}\right)\right]^{-1} \lim _{n \rightarrow \infty} \operatorname{Tr}\{R(D) D \exp (-B / n)} \\
& \left.\times \exp [-R(B / n)] \exp \left[C_{1} R\left(C_{1}\right) / n\right] \cdots\right\}
\end{aligned}
$$

by the Trotter product formula. Now expand each factor $\exp \left[C_{i} R\left(C_{i}\right) / n\right]$ in a power series. Then $\langle R(D) D\rangle$ is a sum of terms of the form $\operatorname{Tr}\left[R\left(Q_{1}\right) Q_{1} \ldots\right.$ $\left.R\left(Q_{n}\right) Q_{n}\right]=\operatorname{Tr}\left[R\left(Q_{1} \cdots Q_{n}\right)\right] \operatorname{Tr}\left(Q_{1} \cdots Q_{n}\right)=\operatorname{Tr}\left(Q_{1} \cdots Q_{n}\right)^{2} \geqslant 0$ since $Q_{1} \cdots Q_{n}$ are real matrices.

Now let $\langle\cdots\rangle_{\infty}$ denote an infinite-volume state obtained as a limit point of the states in volumes with $L_{1}$ even. The state $\langle\cdots\rangle_{\infty}$ is automatically translation invariant since each finite-volume state is. Let $R$ be defined as above, and define an inner product $((\cdot, \cdot))$ on the operators on the sites with $\alpha_{1} \geqslant 1$ by $((A, B))=\left\langle R\left(A^{*}\right) B\right\rangle$. Let $\mathscr{H}$ be the Hilbert space obtained by completing in this inner product. Following the idea of Osterwalder and Schrader, ${ }^{(34)}$ we have the result:

Theorem E.2. Let $\tau_{n}$ be the automorphism on the infinite-volume quasilocal algebra obtained by translating $n$ units to the right. Then, there is a self-adjoint contraction $T$ on $\mathscr{H}$ such that for any operators $A, B$ on the sites with $\alpha_{1} \geqslant 1$

$$
\left(\left(A, \tau_{n}(B)\right)\right)=\left(\left(A, T^{n}(B)\right)\right)
$$

Remark. $T$ is an abstract version of the transfer matrix with the important difference of $n+1$ spacing mentioned above.

Proof. We begin by proving that

$$
\left|\left(\left(A, \tau_{1}(B)\right)\right)\right| \leqslant((A, A))^{1 / 2}((B, B))^{1 / 2}
$$

for $A, B$ bounded. Clearly, $\left|\left(\left(A, \tau_{n}(B)\right)\right)\right| \leqslant\|A\|\|B\|$. Moreover,

$$
\begin{aligned}
\left(\left(A, \tau_{1}(B)\right)\right) & \leqslant((A, A))^{1 / 2}\left(\left(\tau_{1}(B), \tau_{1}(B)\right)\right)^{1 / 2}=((A, A))^{1 / 2}\left(\left(B, \tau_{2}(B)\right)\right)^{1 / 2} \\
& \leqslant((A, A))^{1 / 2}((B, B))^{1 / 4}\left(\left(B, \tau_{4}(B)\right)\right)^{1 / 4} \ldots \\
& \leqslant((A, A))^{1 / 2}((B, B))^{\left(1-2^{-n}\right) / 2}\left(\left(B, \tau_{2^{n+1}}(B)\right)\right)^{2^{-n-1}}
\end{aligned}
$$


Taking $n$ to $\infty$, (E4) results. Thus, there is a contraction $T$ such that $\left(\left(A, \tau_{1}(B)\right)\right)=\left((A, T(B))\right.$. Since $\left(\left(\tau_{1}(A), B\right)\right)=\left(\left(A, \tau_{1}(B)\right)\right), T$ is self-adjoint. Moreover, since $\tau_{n}=\left(\tau_{1}\right)^{n}$, we have (E3).

Corollary E.1. Fix $\alpha_{2}, \ldots, \alpha_{v}$ and an operator $B$ at a single site. Let $g\left(\alpha_{1}\right)=\left\langle B_{\left(0, \alpha_{2}, \ldots, \alpha_{v}\right)}^{*} B_{\left(\alpha_{1}, \alpha_{2}, \ldots, \alpha_{\nu}\right)}\right\rangle$ for $\alpha_{1} \geqslant 1$. Then there is a positive measure $d \mu_{B}$ on $[-1,1]$ such that

$$
g(\alpha)=\int_{-1}^{1} \lambda^{\alpha-1} d \mu_{B}(\lambda), \quad \alpha=1,2, \ldots
$$

Proof. Since $g\left(\alpha_{1}\right)=\left(\left(B_{\left(1, \alpha_{2}, \ldots, \alpha_{\nu}\right)}, T^{\alpha_{1}-1} B_{\left(1, \alpha_{2}, \ldots, \alpha_{\nu}\right)}\right)\right)$, (E5) follows by the spectral theorem.

There is at this point one important difference from the Euclidean field theory case. A semigroup indexed by $\mathbb{R}$ of self-adjoint operators is automatically a semigroup of positive operators. This is not automatic in the discrete case. It is natural to ask if this is so, i.e., if $d \mu_{B}$ in (E5) is supported on $[0,1]$.

\section{ACKNOWLEDGMENTS}

It is a pleasure to thank several individuals for valuable aid: J. F. Barnes and M. L. Glasser for helping find numerical values of various integrals, V. Bargmann for simplifying one of our technical proofs (Theorem A.2), P. A. Vuillermot for valuable discussions, and J. Fröhlich for a variety of useful comments and, in particular, for communicating to us a conjectured bound which we proved (Theorem 4.2) in the real case and used as an important part of our argument. We also thank him for pointing out a serious error in our original manuscript, namely that our "proof" of Theorem 4.2 was wrong in the complex case.

\section{REFERENCES}

1. P. W. Anderson, Phys. Rev. 83:1260 (1951).

2. T. Asano, J. Phys. Soc. Japan 29:350-359 (1970).

3. D. Bessis, P. Moussa, and M. Villani, J. Math. Phys. 16:2318 (1975).

4. F. Bloch, Z. Physik 61:206 (1930).

5. N. Bogoliubov, Phys. Abh. S.U. 1:113-229 (1962).

6. H. Brascamp and E. H. Lieb, in Functional Integration and Its Applications, A. N. Arthurs, ed., Clarendon Press, Oxford (1975).

7. L. Breiman, Probability, Addison-Wesley (1967).

8. R. L. Dobrushin, Teorija Verojatn i ee Prim. 10:209-230 (1965).

9. F. J. Dyson, Phys. Rev. 102:1217 (1956).

10. F. J. Dyson, Phys. Rev. 102:1230 (1956). 
11. R. Ellis, J. Monroe, and C. Newman, Comm. Math. Phys. 46:167-182 (1976).

12. J. Fröhlich, B. Simon, and T. Spencer, Comm. Math. Phys., to appear.

13. J. Ginibre, Comm. Math. Phys. 14:205 (1969).

14. J. Glimm, A. Jaffe, and T. Spencer, Comm. Math. Phys. 45:203 (1975).

15. R. Griffiths, Phys. Rev. 136A:437-439 (1964).

16. R. Griffiths, Phys. Rev. 152:240-246 (1966).

17. R. Griffiths, J. Math. Phys. 8:478-483 (1967).

18. R. Griffiths, Comm. Math. Phys. 6:121-127 (1967).

19. F. Guerra, L. Rosen, and B. Simon, Ann. Math. 101:111-259 (1975).

20. K. Hepp and E. H. Lieb, Phys. Rev. A 8:2517-2525 (1973).

21. P. Hohenberg, Phys. Rev. 158:383 (1967).

22. F. Keffer, in Handbuch der Physik, Band XVIII/2, Springer (1966), pp. 1-273.

23. A. Klein, Bull. Am. Math. Soc. 82:762 (1976).

24. R. Kubo, J. Phys. Soc. Japan 12:570 (1957).

25. E. H. Lieb and D. Mattis, J. Math. Phys. 3:749 (1962).

26. E. H. Lieb and D. Mattis, Phys. Rev. 125:164 (1962).

27. E. H. Lieb and D. W. Robinson, Comm. Math. Phys. 28:251-257 (1972).

28. N. Mermin and H. Wagner, Phys. Rev. Lett. 17:1133 (1966).

29. H. Mori, Prog. Theor. Phys. 33:423 (1965).

30. J. Naudts and A. Verbeure, J. Math. Phys. 17:419-423 (1976).

31. J. Naudts, A. Verbeure, and R. Weder, Comm. Math. Phys. 44:87-99 (1975).

32. C. Newman, in Proc. 1975 Marseille Conference on Quantum Field Theory and Statistical Mechanics.

33. L. Onsager, Phys. Rev. 65:117 (1944).

34. K. Osterwalder and R. Schrader, Comm. Math. Phys. $31: 83$ (1974).

35. R. Peierls, Proc. Camb. Phil. Soc. 32:477-481 (1936).

36. R. Powers, U.C. Berkeley Preprint (1976).

37. M. Reed and B. Simon, Methods of Modern Mathematical Physics, I, Functional Analysis, Academic Press (1972).

38. D. Robinson, Comm. Math. Phys. 7:337 (1968).

39. D. Robinson, Comm. Math. Phys. 14:195 (1969).

40. G. Roepstorf, Comm. Math. Phys. 46:253-262 (1976).

41. D. Ruelle, Statistical Mechanics, Benjamin (1969).

42. H. Samelson, Notes on Lie Algebras, Van Nostrand (1969).

43. T. Schultz, D. Mattis, and E. H. Lieb, Rev. Mod. Phys. 36:856 (1964).

44. R. Streater, Comm. Math. Phys. 6:233 (1967).

45. G. N. Watson, Quart. J. Math. 10:266 (1939).

46. J. Fröhlich, private communication.

47. H. Falk and L. W. Bruch, Phys. Rev. 180:442 (1969).

48. S. Malyshev, Comm. Math. Phys. 40:75 (1975).

49. J. Fröhlich and E. H. Lieb, Phys. Rev. Lett. 38:440-442 (1977); J. Fröhlich and

E. H. Lieb, Comm. Math. Phys., in press.

50. J. Fröhlich, R. Israel, E. H. Lieb, and B. Simon, papers in preparation.

51. F. J. Dyson, E. H. Lieb, and B. Simon, Phys. Rev. Lett. 37:120-123 (1976). 